Check for updates

Cite this: RSC Adv., 2017, 7, 25070

\title{
L-Arginine intercedes bio-crosslinking of a collagen-chitosan 3D-hybrid scaffold for tissue engineering and regeneration: in silico, in vitro, and in vivo studies
}

\author{
Sivalingam Udhayakumar, (D) Krishnakumar Gopal Shankar, Sampath Sowndarya, \\ Sankar Venkatesh, Chellappa Muralidharan and Chellan Rose (D)*
}

Three-dimensional (3D) collagen (COL) scaffolds have recently emerged as functional biomaterials in tissue engineering and wound healing. The limitation includes toxicity of synthetic crosslinkers, weak mechanical stability, low biocompatibility and fast biodegradation. Bovine collagen incorporated with chitosan (COLCS) was cross-linked with amino acids L-arginine (ARG), glutamic acid (GLU) and lysine (LYS) or without amino acids and was fabricated by the freeze-drying method. Morphological characteristics of 3D porous scaffolds observed by SEM showed a uniform interconnected porous structure with pore size ranging from 51 of $450 \mu \mathrm{m}$. Intermolecular interactions between the bio-polymers were examined by FT-IR. Thermal properties using DSC and TGA, swelling ability in PBS $(\mathrm{pH} 7.4)$ and biodegradability by collagenase digestion of the scaffolds were assessed. The amino acid cross-linked scaffolds have greatly improved thermal stability and reduced biodegradation. In vitro fibroblast cultures on the 3D scaffolds proved cyto-compatibility and enhanced proliferative ability. Fluorescence microscopy and SEM were conducted to examine cell morphology and its attachment on the scaffolds. Cell viability was also confirmed by using flow cytometry. The overall result authenticates that the ARG cross-linked scaffold (COL-CS-A) augmented cellular growth compare to other cross-linked scaffolds followed by uncrosslinked scaffolds (control). In silico studies also proved the fact that ARG can act as a suitable cross-linker for collagen and chitosan complex systems. Moreover, the in vivo wound healing studies in a rat model revealed that the COL-CS-A dressing promotes the healing and accelerates the re-epithelialization and collagen deposition. Overall, the findings confirm that the COL-CS-A hybrid scaffold was an excellent candidate for tissue engineering and also for regenerative medicine.

Received 8th March 2017

Accepted 21st April 2017

DOI: $10.1039 / \mathrm{c} 7 \mathrm{ra02842c}$

rsc.li/rsc-advances

\section{Introduction}

Tissue engineering is a new strategy for the development of new biological substitutes, also called biomaterials or scaffolds to maintain or restore the function of damaged/diseased tissues and organs. ${ }^{1,2}$ In recent years there has been an increasing interest in using 3D porous scaffolds as cell culture models in order to study cell-matrix interactions and their effects on cell function as they provide a 3D structure for cell adhesion, proliferation, differentiation, secretion of extracellular matrices, and physical support for new tissue formation and regeneration. ${ }^{3,4}$ To achieve these biological functions, scaffolds should be biocompatible with the host, must be biodegradable during tissue growth, must have completely interconnected pores to transport nutrients and facilitate cell colonization/biosynthesis

Department of Biochemistry and Biotechnology, CSIR-Central Leather Research Institute, Chennai 600020, India. E-mail: chellanrose@yahoo.co.uk; chellanrose88@ gmail.com; Tel: +91-44-24430273 and to provide appropriate mechanical strength to maintain structural integrity and tissue formation., ${ }^{5,6}$ The engineering of scaffolds is usually composed of natural and/or synthetic polymers for tissue engineering. Natural materials including collagen, chitosan and hyluronic acid and synthetic biopolymers, such as poly-lactic acid (PLA), poly-lactic-co-glycolide (PLGA), poly-glycolic acid (PGA) and poly-caprolactone (PCL ${ }^{7-10}$ were widely used in scaffold fabrication. The complete utilization of these materials for scaffold preparation was restricted because of low mechanical strength and biocompatibility with cells.

Natural polymers such as collagen and chitosan, are widely used as the main component for scaffold preparation. Collagen is a fibrous protein and it has diverse applications in the biomedical field for its excellent biocompatibility, biodegradability and low antigenicity. ${ }^{11,12}$ It plays a major role in the formation of extracellular matrix and in controlling cell behaviors such as adhesion, proliferation and differentiation in the development/healing of tissue and organs. ${ }^{13-15}$ However, the low mechanical strength and the fast biodegradation rate of 
collagen are adverse factors that limit the use of this material. Various modified technologies have been applied to increase the stability of collagen and to control its degradation rate. In particular, collagen may be mixed with other viable polymer material to increase mechanical properties. ${ }^{16}$ Chitosan is the second most abundant natural biopolymer used in a variety of biomedical fields such as tissue regeneration, drug delivery carriers, surgical thread, and wound healing materials. ${ }^{17}$ It is a natural polysaccharide that is structurally similar to GAGs and consists of $\beta$-(1-4) linked $\mathrm{D}$-glucosamine residues with a variable number of randomly located $N$-acetyl-glucosamine groups. It has been reported to be biocompatible, nontoxic, and to promote wound healing. ${ }^{18}$ In addition, the combination of collagen with chitosan is known to increase mechanical strength and the biological property of scaffold than the individual material, ${ }^{6}$ as it forms an ionic complex between negatively charged collagen and the positively charged chitosan molecule. ${ }^{19}$ Furthermore, it exhibits antimicrobial properties such as antibacterial ${ }^{20}$ and antifungal ${ }^{21}$ activities along with mucoadhesive, ${ }^{22}$ haemostatic properties, ${ }^{23}$ minimal foreign body reactions and increased cell infiltration. ${ }^{24}$

In recent years, various methods are engaged to develop the physical and biological properties of scaffolds made of polymeric materials. Cross-linking of polymer is one such method to optimize mechanical stability and to effectively slow down the biodegradation rate of the collagen material. ${ }^{25}$ Therefore, the cross-linking of collagen materials has become one of the essential concerns for the collagen scaffolds with porous nature. Currently, there are two types of cross-linking techniques frequently employed in scaffold fabrication, such as physical methods and chemical methods. The former method deals with the use of photo-oxidation, dehydrothermal treatments (DHT) and ultraviolet irradiation, which prevents the formation of potential cytotoxic chemical residuals and maintains the biocompatibility of collagen biomaterials. ${ }^{26}$ Physical treatments are fails to yield adequate cross-linking degree to meet the requirements of tissue engineering application. This can be overcome by the chemical methods where glutaraldehyde (GA) is the most frequently used traditional cross-linker in the collagen cross-linking studies, as it can enhance the biological stability of the scaffolds. However, some problems arise in the utilization of GA, such as over cross-linking and the potential cytotoxicity. ${ }^{27,28}$ Therefore, nontoxic and biocompatible cross-linking agents such as amino acids are now being considered for cross-linking in scaffold preparation. ${ }^{29}$

Currently, there are a variety of wound dressings commercially available that are ranging from passive adherent/nonadherent to bioactive products that facilitate the healing process. Collagen dressings that are mostly fabricated as gels (Collasate ${ }^{\circ}$, PRN Pharmacal, Pensacola, FL), porous and nonporous membranes (AVITENE ${ }^{\mathrm{TM}}$ Davol, A Bard Company, Warwick, RI), particles (CollamendTM Veterinary Products Laboratory, Phoenix, AZ) and sponges (SpongeCol ${ }^{\circledR}$, Advanced BioMatrix, Inc. San Diego, CA) are reportedly improves wound healing in humans and animals. Even with considerable advancements in wound healing dressings, it shows that there is no single material can produce the optimum microenvironment for all wounds or for all the stages of the wound healing process. ${ }^{30-32}$ Therefore the development of improved collagen dressings containing bioactive polymers is indispensable for wound management.

It has been reported that the daily change of hydrolyzed bovine collagen dressing on open wounds, cleaned the contaminated wounds. ${ }^{33}$ After 7 days of treatment wound significantly enhanced the epithelialization compared with a semiocclusive nonadherent control dressing. Yoo Jung Park et al. used type-I collagen dressing in the treatment of diabetic foot ulcer and dressing changes had been performed two or three times per week where the study group showed a higher rate of complete healing than the control group. ${ }^{34}$ The gel containing a collagen with chitosan has been reported to enhance the healing rate of cutaneous excisional wound compared to the individual biopolymer alone..$^{35}$ Likewise, the most metabolically versatile amino acid L-arginine has been reported to posses many physiologically active roles within the human body, as it is essential for the growth of a wide variety of cells. $^{36}$ Increased collagen deposition and wound breaking strength $^{37}$ have an effective anti-aging, ${ }^{38}$ anti-oxidant activity ${ }^{39}$ and immune modulatory effect ${ }^{40}$ that promote cell growth and its differentiation. L-Arginine has also been reported to enhance cell proliferation and wound healing response by stimulating $\mathrm{T}$ cell responses, which then increases fibroblast responses ${ }^{41,42}$ and has applications in the treatment of wounds. However, the L-arginine in combination with bovine collagen (COL) and chitosan (CS) 3D hybrid scaffold has not been reported in tissue engineering.

In consideration of the biologically active properties of natural materials, we prefer to use -arginine (ARG) in this study as a cross-linker and an active component to prepare a biocompatible COL-CS 3D scaffold in order to improve the physical, thermal, and biological properties of the porous scaffold for tissue engineering applications. The ARG was comparatively studied with amino acids such as L-glutamic acid (GLU) and L-lysine (LYS) to evaluate its superiority in tissue engineering. We developed COL-CS 3D porous scaffold incorporated with amino acid using the freeze-drying method and characterized by using SEM, FT-IR, TGA and DSC. In addition to this, we evaluated the biocompatibility of this novel $3 \mathrm{D}$ hybrid scaffolds in vitro by using NIH3T3 mouse fibroblasts and also monitored by flow cytometry. The possible binding interactions in COL-CS-A compounds were studied in silico. The porous 3D scaffold of this study, after dipping in PBS was applied on to the wound surface, as has been reported in our previous study where 2D collagen film served to cover the surface of the excision wound. ${ }^{43}$

\section{Materials and methods}

\subsection{Materials and reagents}

L-Arginine, L-glutamic acid, L-lysine, chitosan ( $80 \%$ deacetylation), Dulbecco's Modified Eagle's Medium-L glucose (DMEM), $0.25 \%$ trypsin-EDTA solution, $100 \times$ antibiotic and antimycotic solution, MTT [3-(4,5-dimethylthiazol-2-yl)-2,5-diphenyl tetrazolium bromide], dimethyl sulphoxide (DMSO), acridine orange 
(AO), ethidium bromide (EB), glutaraldehyde, sodium cacodylate, osmium tetroxide, potassium bromide ( $\mathrm{KBr})$, collagenase (Clostridium histolyticum) were obtained from Sigma Aldrich (St. Louis, MO, USA). Fetal Bovine Serum (FBS) was purchased from Pan Biotech Company, Germany. Annexin V-FITC apoptosis detection kit was obtained from eBioscience, USA. The NIH-3T3 (mouse fibroblast) cell line was procured from National Centre for Cell Sciences, Pune, India. All other chemicals and reagents used were of analytical or molecular grade.

\subsection{Extraction of collagen}

Soluble type I collagen was extracted from the tissue of bovine fore-stomach (domestic cattle of Indian origin). The freshly obtained tissue was thoroughly washed in cold water prior to chopping into small pieces and minced by using meat mincer (ADE FL-E 2200, Germany). Tissue treated with $0.1 \%(\mathrm{w} / \mathrm{w})$ pepsin in acidic medium, pH 2.0 at $4{ }^{\circ} \mathrm{C}$ for $24 \mathrm{~h}$ was filtered and the filtrate was neutralized using $1 \mathrm{~N} \mathrm{NaOH}$; then collagen was precipitated by the addition of $\mathrm{NaCl}(30 \% \mathrm{w} / \mathrm{w})$. The pellet was then collected by centrifugation at $10000 \mathrm{rpm}$ for $20 \mathrm{~min}$ at $4{ }^{\circ} \mathrm{C}$ and resuspended in $0.5 \mathrm{M}$ acetic acid. After dialysis against deionized water followed by $0.2 \mathrm{M}$ disodium hydrogen phosphate, the dialysate was centrifuged and the pellet was then dispersed in $0.5 \mathrm{M}$ acetic acid. The solution was further dialyzed against $0.05 \mathrm{M}$ acetic acid and the resultant collagen solution was stored at $-20{ }^{\circ} \mathrm{C}$ until required.

\subsection{Scaffold fabrication}

The 3D scaffolds were prepared by freeze-drying technique. Collagen solution was prepared with $2 \%(\mathrm{w} / \mathrm{v})$ collagen in $0.1 \mathrm{M}$ acetic acid at $-4{ }^{\circ} \mathrm{C}$. After overnight agitation, the solution was filtered using a filter cloth in order to remove any insoluble particles. To fabricate bioactive 3D scaffold, the chitosan (2\%) were dissolved in $0.1 \mathrm{M}$ acetic acid and mixed with collagen (2\%) in the ratio of $1: 1$. The mixture was blended for $15 \mathrm{~min}$ using a hand blender (Philips HR 1063, India). The COL-CS blend was then instantly cross-linked with L-arginine, L-glutamic acid or L-lysine at a fixed concentration of $2.5 \mu \mathrm{M}$, by directly mixing each amino acid separately to the hybrid solution and kept in stirrer for $24 \mathrm{~h}$ at $-4{ }^{\circ} \mathrm{C}$. The resulting viscous sample was then poured into the cavities of 24 well plate $(2 \mathrm{~mL}$ per well) and $60 \mathrm{~mm}^{2}$ Petri dish and deep frozen at $-80{ }^{\circ} \mathrm{C}$ for $24 \mathrm{~h}$ and then lyophilized (Operon Co., Korea). Similarly, the solution without amino acids was also lyophilized and all the scaffolds were kept in air-tight plastic containers and stored in $-20{ }^{\circ} \mathrm{C}$ for further experimental use.

\subsection{Micro structure examination}

The 3D architecture, surface morphology and pore structure of the freeze-dried scaffolds were inspected using scanning electron microscopy (HR-SEM, FEI; Quanta FEG 200, USA). Thin sections of the samples were sputter coated with a thin layer of gold ions to an approximate thickness of $200 \AA$ using Polaron Sputtering apparatus and both surface and cross section were analyzed. Triplicates of all samples were examined and the average pore diameter of each scaffold was plotted using Image-
J software. The porosity of the scaffold was determined by the ethanol displacement method and calculated by using the formula, $p=\left(v_{1}-v_{3}\right) /\left(v_{2}-v_{3}\right)$ where $p$ is the porosity, $v_{1}$ is the initial volume of ethanol, $v_{2}$ indicates volume after scaffold immersion and $v_{3}$ is volume after scaffold removed from ethanol.

\subsection{Infrared (IR) spectroscopic analysis}

Fourier-transformed infrared (FT-IR) spectrum of the individual samples was taken to determine the functional groups present in the prepared scaffolds by using a Perkin-Elmer (USA) FT-IR spectroscope. Each freeze-dried scaffold sample was ground into fine powder and embedded in $\mathrm{KBr}$ in the pellet form. The samples in $\mathrm{KBr}$ pellet were screened in the wavelength range between 400 and $4000 \mathrm{~cm}^{-1}$ at a resolution of $0.7 \mathrm{~cm}^{-1}$.

\subsection{Thermal characterization}

The thermal transition was analyzed by the differential scanning calorimetric (DSC) method. The experiment was carried out by using DSC the device (Q200 V23.10, USA) calibrated with indium. The scaffolds weighing about $5 \mathrm{mg}$ were used and heated from $30{ }^{\circ} \mathrm{C}$ to $300{ }^{\circ} \mathrm{C}$ with a heating rate of $10{ }^{\circ} \mathrm{C} \mathrm{min}{ }^{-1}$ in a nitrogen atmosphere. The endothermic peak temperature shifts between the scaffolds were investigated.

Thermogravimetric analysis (TGA) was performed using TA instrument series (TA-2950, USA) and results were interpreted by using thermogravimetric analyser software (Universal Analysis 2000). For TGA, $5 \mathrm{mg}$ of samples were used and scanned at $10{ }^{\circ} \mathrm{C} \mathrm{min}^{-1}$. The experiments were performed under a nitrogen atmosphere $\left(50 \mathrm{~mL} \mathrm{~min}^{-1}\right)$ and denaturing temperature was increased from 25 to $800{ }^{\circ} \mathrm{C}$. The experiments were conducted in triplicates for each scaffold. The TGA results were plotted by using the OriginPro 8.5 software package.

\subsection{Swelling test}

The water uptake ability of the 3D scaffolds was analyzed according to the method followed by Adekogbe and Ghanem (2005). ${ }^{44}$ The samples were cut into small pieces with equal dry weights and separately immersed in 0.1 M PBS, pH 7.4, at room temperature. The soaked samples were removed after the determined time interval, blotted on filter paper to remove excess water and weighed. The initial and final weights of scaffolds were noted as $w_{0}$ and $w_{t}$ and percentage of water uptake was determined by using the formula.

$$
\text { Percentage of water uptake }(\%)=100 \times\left(w_{t}-w_{\mathrm{o}}\right) / w_{\mathrm{o}}
$$

\subsection{Mechanical properties}

The tensile strength of the 3D scaffolds was measured in dry state using a universal testing machine (INSTRON VTT 3369, Finland) with a load cell of $100 \mathrm{~N}$, and the speed of crosshead was about $5 \mathrm{~mm} \mathrm{~min}^{-1}$. The distance between the two air clamps was set to $20 \mathrm{~mm}$. The scaffolds were cut into dumbbellshaped strips and were measured using a digital caliper micrometer (Ultra CAL IV; Ted Pella, Inc. USA). To determine 
the sample size, four different regions of the sample were measured and averaged. The five samples of each scaffold with the size of $4 \mathrm{~cm}$ length and $4 \mathrm{~mm}$ width were clamped and tested at a temperature of $20{ }^{\circ} \mathrm{C}$ with relative humidity of $65 \%$. The average value of tensile strength $(\mathrm{MPa})$, elongation at break and Young's modulus were evaluated.

\subsection{In vitro collagenase degradation}

The rate of degradation and biological stability of the scaffolds were evaluated by in vitro collagenase enzyme biodegradation test as described by Pieper JS et al. $1999 .{ }^{45}$ All samples with equal weight were immersed in phosphate buffered saline (PBS, $\mathrm{pH}$ 7.4) medium containing collagenase enzyme (100 $\mathrm{U} \mathrm{mL}^{-1}$ ) and incubated at $37{ }^{\circ} \mathrm{C}$ for 6 days. PBS was used in this experiment, as it was isotonic to animal cells and prevented the denaturation of proteins. Degradation of samples was discontinued at the given time interval by incubating in ice bath, followed by centrifugation at $1500 \mathrm{rpm}$ for $10 \mathrm{~min}$. Supernatant was hydrolyzed with $6 \mathrm{M} \mathrm{HCl}$ at $120{ }^{\circ} \mathrm{C}$ for $12 \mathrm{~h}$. The content of released hydroxyproline from the material was measured with ultraviolet spectroscopy at a wavelength of $\lambda=560 \mathrm{~nm}$. The biodegradation degree is defined as the percentage of hydroxyproline released from the samples at different times to the completely degraded one with the same composition and weight.

\subsection{Cell culture}

NIH-3T3 cells was cultured in DMEM-L glucose, supplemented with $10 \%$ FBS, $0.1 \%$ antibiotic-antimycotic solution, at $37{ }^{\circ} \mathrm{C}$ with $95 \%$ humidity in $5 \% \mathrm{CO}_{2}$ incubator (NuAire, MN, USA). The culture medium was changed every 2 to 3 days. For cell culture experiments, circular shaped cross-linked collagen scaffolds with $1 \mathrm{~cm}^{2}$ area and $2 \mathrm{~mm}$ thickness were used. Prior to cell seeding, the scaffolds were sterilized using $0.01 \%$ antibiotic/antimycotic solution in $70 \%$ ethanol for $12 \mathrm{~h}$, extensively washed thrice in sterile PBS for $30 \mathrm{~min}$, and then sterilized under UV radiation for $2 \mathrm{~h}$. Samples were soaked in fresh medium for $24 \mathrm{~h}$ to check culture sterility. Then, the scaffolds were placed in a 24 well plate and aliquots of NIH 3 T3 fibroblast cell suspension $\left(3 \times 10^{5}\right.$ cells per well) were cultured in scaffolds for a predetermined period. The cells cultured in 24 well plates without scaffold were used as control. The cytotoxicity, biocompatibility and cell morphology were analyzed.

\subsection{Cytotoxicity assay}

Cytotoxicity of the scaffolds were determined using the MTT (3dimethylthiazol-2,5-diphenyltetrazolium bromide) colorimetric assay. Cells cultured in 24 well plates were incorporated with or without scaffold was incubated for 24,48 , and $72 \mathrm{~h}$. The cells containing scaffold were then stained with $0.5 \mathrm{~mL}$ of MTT solution $\left(0.5 \mathrm{mg} \mathrm{mL}^{-1}\right.$ in DMEM without phenol red) at $37{ }^{\circ} \mathrm{C}$ for 2 to $3 \mathrm{~h}$. Mitochondrial enzymes of viable cells reduced the yellow-colored MTT into purple-colored formazan crystals; it was then dissolved in $200 \mu \mathrm{L}$ of DMSO for $15 \mathrm{~min}$. Absorbance of the solubilized formazan derivative was measured at $\lambda=$ $540 \mathrm{~nm}$ using the Microplate reader (Thermo Scientific
Multiskan Ascent, USA) to determine the number of living cells. Finally, the cell viability of scaffolds was compared with the controls and percentage of viability was calculated using the formula:

$$
\% \text { viability }=(\mathrm{OD} \text { of test sample/OD of control }) \times 100 .
$$

\subsection{Cell attachment and proliferation studies}

The morphology and attachment of NIH-3T3 fibroblasts on the scaffolds after $48 \mathrm{~h}$ of culture were monitored using SEM and the fluorescent microscope. In brief, the scaffolds with cells were washed with PBS to remove non-adherent cells, and fixed with $2.5 \%$ glutaraldehyde at $4{ }^{\circ} \mathrm{C}$ for $4 \mathrm{~h}$. Afterwards, the samples were dehydrated using a series of ethanol solutions (25, 50, 75, 95, and $99 \mathrm{wt} \%)$. Dried cell-scaffold constructs were sputter coated with a thin layer of gold and the morphology of cells on the scaffolds was observed by using SEM (FEI; Quanta FEG 200, USA). The proliferation of the cells cultured in the scaffold was analyzed by using a fluorescent stain-containing mixture of AO and EB, both $100 \mu \mathrm{g} \mathrm{mL}^{-1}$ in PBS $1: 1$ ratio (v/ v). After 5 min incubation, the cells were visualized under the fluorescence microscope (LEICA-DM IL LED FLUO, Germany).

\subsection{Flow cytometry}

Cell viability was quantified by flow cytometry. In brief, $3 \times 10^{5}$ cells per well were cultured on scaffolds, after $48 \mathrm{~h}$ cells were trypsinized and resuspended in original media and washed with PBS. Cells were then labelled with Annexin V-FIT C and propidium iodide (PI) according to manufacturer's instructions. Cell population was read on the FL-1 channel of Becton Dickinson immunocytometry systems; the FACS Calibur cytometer, was validated by using Cell Quest Pro software.

\subsection{Molecular docking studies}

Molecular docking was carried out using molecular docking suite AutoDock Vina 1.1.2. Fasta sequence of collagen like peptide was fetched UniProt (P02452). ${ }^{46}$ We took two models with (EVGPPGPPGPAGEKGSPGADGPAGAPG) $)_{3}$ and (TPG PQGIAGQRGVVGLPGQRGER) ${ }_{3}$, and generated the 3D structure of a collagen-like peptide using gencollagen (http://www.cgl.ucsf.edu/ cgi-bin/gencollagen.py). The L-arginine (CID 6322) and chitosan (CID 71853) were optimized using the eLBOW module in Phenix software, with the method of UHF and AM1 was selected as the default setting. ${ }^{47,48}$ Collagen like-peptide and both compounds were prepared in the pdbqt format using Auto dock tools. ${ }^{49}$ For studying the molecular interaction, for both models a grid box parameter having coordinates center $\_x=0.01$, center $y=0.01$, center $z=$ 110.818 , size $\_x=40$ size $\_y=40$, size_z $z=126$ with spacing $0.375 \AA$ was set and this portion was retained to interact with L-arginine and chitosan compound. All structural images were generated using PyMol. ${ }^{50}$

\subsection{In vivo animal experiments}

In vivo animal studies were approved by the Institutional Animal Ethics Committee (Reg. No: 466/CPCSEA dated: 
15/2016(a)). Fifteen Wistar albino rats were obtained from the Tamilnadu Veterinary and Animal Sciences University. Animals with $150 \mathrm{~g}$ of weight were equally divided into three groups (five rats per group). On the day of wounding, the rats were anaesthetized by mild ether, and a full thickness excision wound $(1.5 \mathrm{~cm} \times 1.5 \mathrm{~cm})$ was created on the back of each rat. The wounds thus created in two groups were firmly covered with the COL-CS and COL-CS-A respectively. Wounds without scaffold dressing were treated with PBS pH 7.4 and kept as negative control. The dressing materials were changed at every 4 days (if the dressing material was not adhering to the wound surface) and the area of the wound was measured, photographed and the post-wounding granulation tissue was excised and subjected to the quantification of hydroxyproline, ${ }^{51}$ hexosomaine, ${ }^{52}$ and uronic acid ${ }^{53}$ content. Additionally, the tensile strength of the re-epithelialised skin tissues was also measured to determine the degree of deposition of collagen in the healed region.

The healing rate was calculated by using the following formula, where $A_{0}$ is the initial wound area, and $A_{t}$ is the wound area on the day of the changing of dressing.

$$
\% \text { healing rate }=\left[A_{0}-A_{t}\right] / A_{0} \times 100
$$

For the histological examination of wounded skin tissue, samples were excised on days 4, 8, 12 and 14 post-wound and then gently fixed with $10 \%$ formalin. Afterwards, tissue sections were stained with hematoxylin-eosin (H\&E) and Masson's Tricrome (MT) independently and the slides were observed under the bright field microscope (LEICA-DM IL LED, Germany). The inflammatory cells, fibroblast, collagen fibers, neo epidermis and new blood vessels were examined.

\subsection{Statistical analysis}

All the experiments were done in triplicates and quantitative data are presented as mean \pm standard deviation (SD). Statistical analyses were done using one-way ANOVA with Bonferroni post-tests and the means were separated by using GraphPad Prism Software Inc, (Version 6.0, CA, and USA). In all analyses, $P$ $<0.05$ was considered to indicate statistical significance.

\section{Results and discussion}

In the current study, a three-dimensional cross-linked COL-CS porous scaffold was fabricated using the lyophilization technique and its physico-chemical properties were studied. The cellular attachment to the material, cell growth and proliferation in a $3 \mathrm{D}$ environment was evaluated to engineer a tissue construct using fibroblasts. Prior to this, amino acids such as $\mathrm{L}^{-}$ arginine (ARG), glutamic acid (GLU) and lysine (LYS) were crosslinked with the COL-CS biopolymer blend. The $-\mathrm{NH}_{2}$ and $-\mathrm{COOH}$ groups of L-arginine binds with $-\mathrm{COOH}$ group of collagen and $-\mathrm{NH}_{2}$ group of chitosan, respectively through amide bond formation that releases $\mathrm{H}_{2} \mathrm{O}$ molecule. The process of L-arginine-intercede cross-linking between collagen and chitosan molecules is represented in Scheme 2. The scaffold containing fixed concentrations of different amino acids was analyzed to compare the tensile property variation with respect to the pore size of the scaffolds. In this study we aimed at finding the possible application of an amino acid as a crosslinking component for the COL-CS system.

\subsection{Microstructural characteristics}

The microstructure and pore arrangement of scaffolds plays a major role in cellular attachment, migration, proliferation and function in tissue engineering. ${ }^{54}$ SEM images of surface and cross sectioned scaffolds of COL-CS, COL-CS-A, COL-CS-G and COL-CS-L are presented in Fig. $1 \mathrm{~B}$ and C. The SEM images revealed the poorly interconnected pores of COL-CS and COLCS-G but uniform and well interconnected pores in the case of COL-CS-A and COL-CS-L. The pore size of the COL-CS was in the range of $200-370 \mu \mathrm{m}, 70-200 \mu \mathrm{m}$ for COL-CS-A, 30-100 $\mu \mathrm{m}$ for COL-CS-G and 50-250 $\mu \mathrm{m}$ for COL-CS-L. The mean pore size of COL-CS was $256 \pm 25 \mu \mathrm{m}$ and sizes of $110.1 \pm 25,81.6 \pm 31.2$, $156.6 \pm 48 \mu \mathrm{m}$ respectively for three different amino acid crosslinked scaffolds viz COL-CS-A, COL-CS-G and COL-CS-L (Fig. 1D). The results revealed that the cross-linking with aminoacids altered the microarchitecture of the scaffold. The decreased mean pore size and increased porosity were observed in all the aminoacid incorporated scaffolds. The pore size varied depending upon the amino acids used as cross-linker. Each amino acid interacted with the collagen itself or between the collagen and chitosan in its own fashion based on the individual amino acid's chain length, its reactive functional groups, their interactive partnering groups in collagen or chitosan, the electrostatic charges or other bridging forces likely to be contributed by the biological macromolecules and the bridging amino acids.

In tissue engineering, porosity is a crucial factor that increases the surface area for cellular attachment and multiplication which is an essential criterion for bioactive scaffolds. ${ }^{55}$ Amino acid treatment influenced the porosity of the final crosslinked scaffolds. According to Fig. 1D, the porosity of scaffolds was estimated to be $82 \%$ for COL-CS followed by $95 \%, 80 \%$ and 93\% for COL-CS-A, COL-CS-G and COL-CS-L respectively. It is obvious from these values that the basic amino acids L-arginine and L-lysine cross-linking provide better porosity compared to an acidic amino acid such as L-glutamic acid. In addition to pore size and its uniformity, porosity also determines the tensile properties and the water uptake ability of the scaffold that leads to the easy absorption of the medium to facilitate the cells for migration, adherence and proliferation.

\subsection{FT-IR}

FT-IR spectroscopy is an effective technique used to confirm the functional groups of each compound in the scaffold. The FT-IR spectra of ARG, COL-CS and COL-CS-A scaffolds were acquired separately and evidence for cross-linking and possible interaction between polymer and amino acids are depicted in Fig. 2A. The FT-IR spectra of COL-CS and the interaction between type I collagen and chitosan was discussed in detail in our previous report by Jithendra et al. $2013 .^{4}$ 
(A)
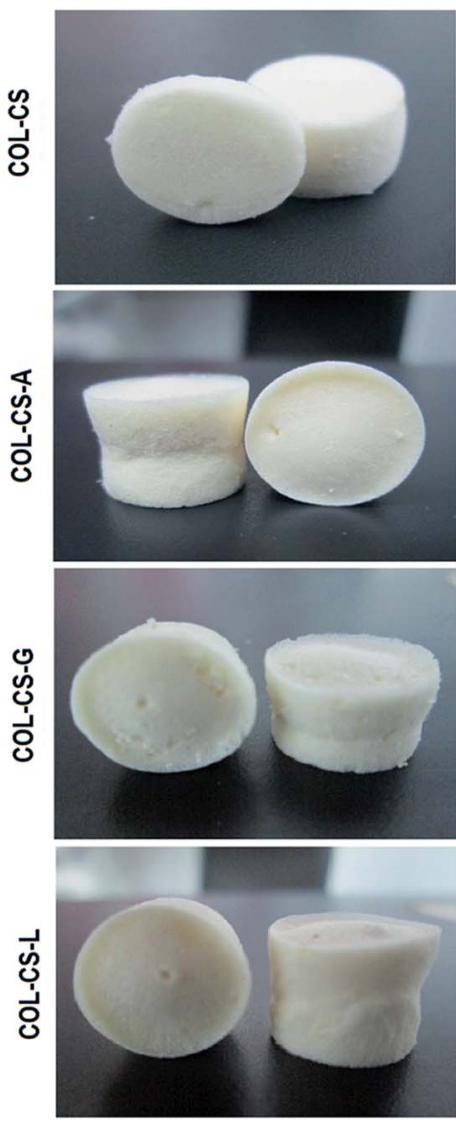

(B)
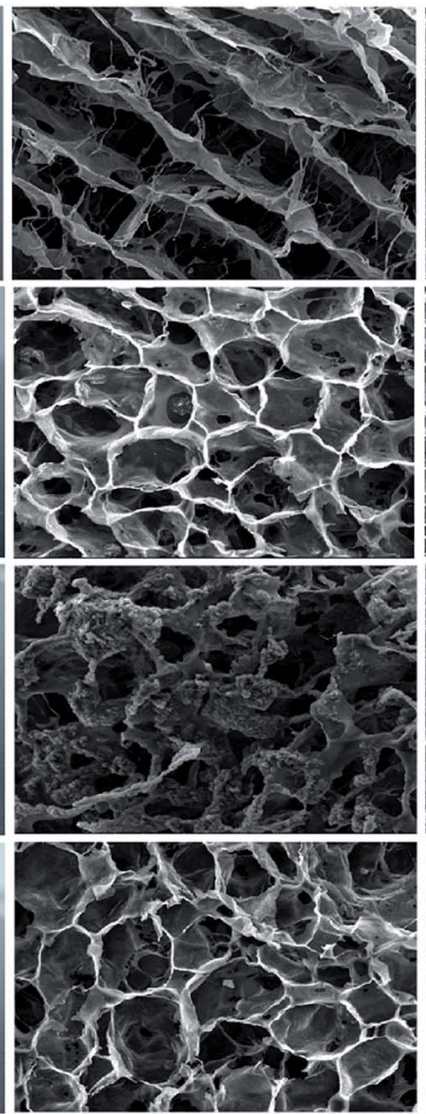

(C)

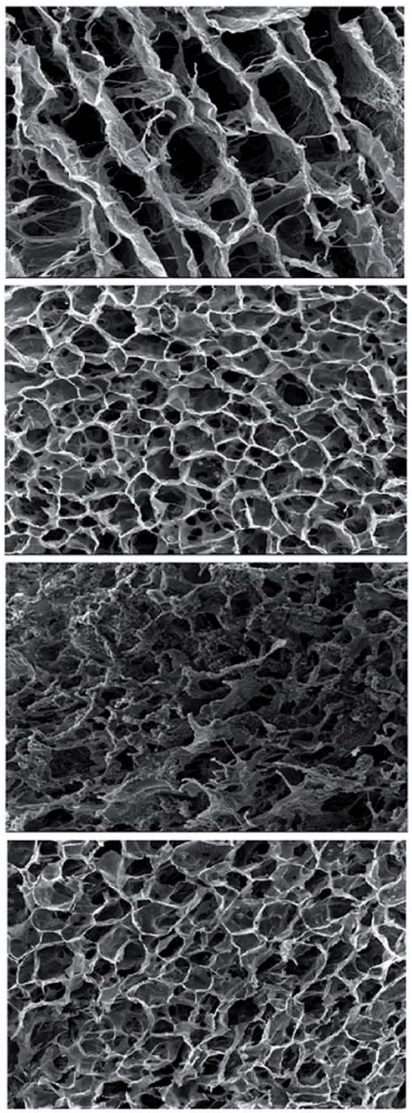

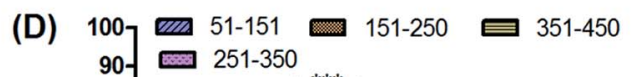

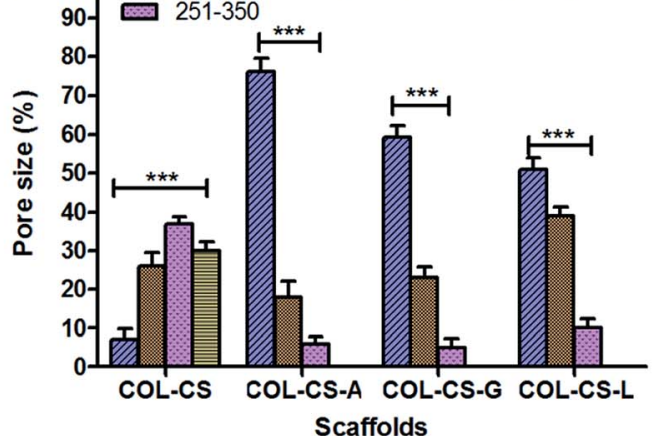

Fig. 1 Photographs of 3D scaffolds (A), SEM images of microstructure of porous scaffolds, surface view (B), cross sections (C), and pore size distribution of scaffolds from the cross-sections (D). Error bars represent the mean $\pm \mathrm{SD}(n=3$; *denotes statistically significant difference, ***p $<0.001)$

The spectrum of COL-CS-A displayed a band shift from 3326 $\mathrm{cm}^{-1}$ to $3263 \mathrm{~cm}^{-1}$, which could be due to the hydrogen bond formation between the carboxyl/amino group of arginine and the $-\mathrm{NH}_{2}$ and $-\mathrm{OH}$ groups in collagen and chitosan. The absorbance peak at $1639 \mathrm{~cm}^{-1}$ is possibly due to amide or ester linkage between the amino group of arginine and the carboxyl group of collagen. The $-\mathrm{OH}$ bend of arginine is shifted from $1536 \mathrm{~cm}^{-1}$ to $1523 \mathrm{~cm}^{-1}$ in COL-CS-A scaffold and this may be due to the effect of arginine. In addition, a peak observed at $2915 \mathrm{~cm}^{-1}$ was due to the asymmetric stretching of the $-\mathrm{CH}_{3}$ group of arginine which was absent in the COL-CS spectra. The absorption peak at $1667 \mathrm{~cm}^{-1}$ due to $\mathrm{NH}_{2}$ bend in arginine was absent in COL-CS spectra. These results reveal that the incorporation of arginine with collagen and chitosan did not accomplish a significant alteration in the chemical property of the polymers but serves as a bridge between a two biomolecules and support the stabilization of this complex hybrid. Accordingly the material can be allowed to exhibit their characteristics independently using in vitro studies, where the cells could interact with their respective components. 
(A)

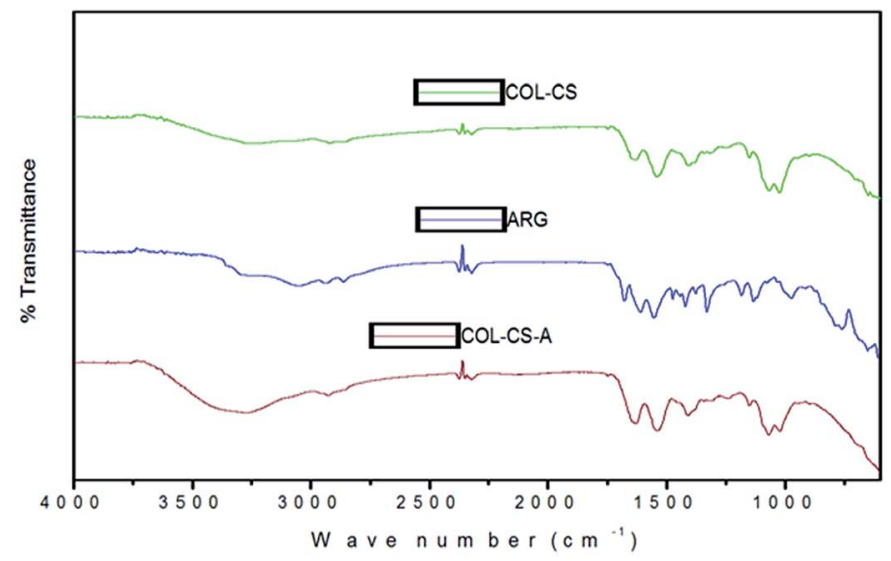

(B)

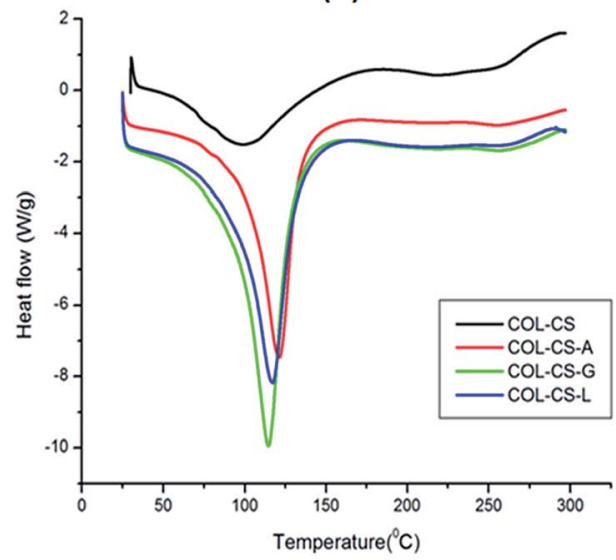

(C)

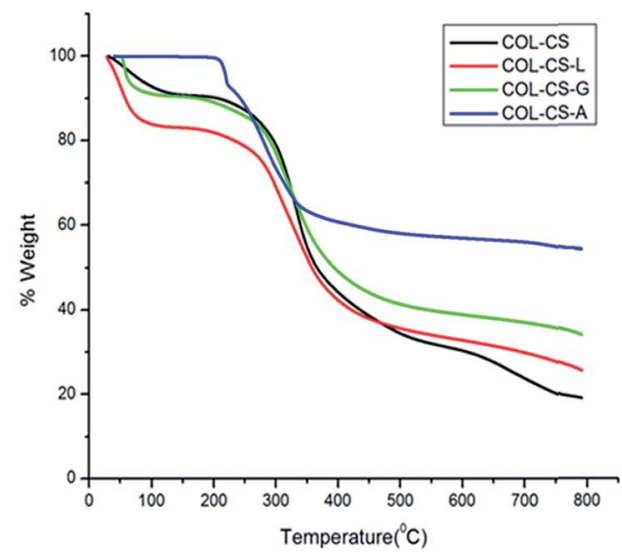

Fig. 2 (A) FTIR spectra of COL-CS, ARG and COL-CS-A showed evidence for cross-linking and possible interaction between polymer and amino acids. (B) Differential scanning calorimetric (DSC) and (C) thermogravimetric (TGA) analysis of the scaffolds COL-CS, COL-CS-A, COLCS-G and COL-CS-L.

\subsection{Thermodynamic property}

The DSC curves of COL-CS, COL-CS-A, COL-CS-G and COL-CSL scaffolds are presented in Fig. 2B. The temperature of dehydration $\left(T_{\mathrm{D}}\right)$ of each scaffold in the nitrogen atmosphere is depicted by respective endothermic peaks. Basically, hydroxyproline plays a major role in the stabilization of the collagen triple-helix structure due to its hydrogen-bonding ability through its $-\mathrm{OH}$ group. ${ }^{56}$ Table 1 shows the enthalpy of dehydration $\left(\Delta H_{\mathrm{D}}\right)$ and characteristic $T_{\mathrm{D}}$ values calculated from the DSC measurements. The DSC results demonstrate that the arginine cross-linked scaffold showed an increased $T_{\mathrm{D}}$ (101.96 $\left.{ }^{\circ} \mathrm{C}\right)$ value compared to scaffolds cross-linked with glutamic acid

Table 1 Thermal properties of different scaffolds

\begin{tabular}{lcccl}
\hline & \multicolumn{3}{l}{$3 \mathrm{D}$ scaffolds } & \\
\cline { 2 - 5 } Thermal property & COL-CS & COL-CS-G & COL-CS-L & COL-CS-A \\
\hline$T_{\mathrm{D}}\left({ }^{\circ} \mathrm{C}\right)$ & 93.10 & 93.60 & 95.55 & 101.96 \\
$\Delta H_{\mathrm{D}}\left(\mathrm{J} \mathrm{g}^{-1}\right)$ & 545.30 & 550.5 & 477.9 & 533.1
\end{tabular}

or lysine. This difference may be attributed to the formation of somewhat more rigid linkage contributed by the guanidine group of arginine. Apart from this the scaffold samples exhibited a remarkable difference between the scaffolds depending upon their individual molecular composition and structural conformation contributed by the cross-linking amino acids.

The resultant increase in the $T_{\mathrm{D}}$ values was in the order of COL-CS-A $>$ COL-CS-L $>$ COL-CS-G $>$ COL-CS. The change in $T_{\mathrm{D}}$ depends on the variation in the molecular weight of the biopolymer composite cross-linked using different amino acids. According to the result of Table 1, the COL-CS-A showed the highest $T_{\mathrm{D}}$ value because of better interaction of arginine between collagen and chitosan and enhancement of the molecular weight of the complex biopolymer. The ARG crosslinked COL-CS scaffold required lower energy than that of COL-CS that is represented by very narrow endothermic peak, which indicates the more susceptible nature of the crosslinking. DSC results also indicated the shift in the temperature of dehydration values of COL-CS in the presence of ARG. This certainly showed that ARG cross-linking increased the resistance to pyrolysis and provides stability to the material. The 
decreased $\Delta H_{\mathrm{D}}$ values of COL-CS-A compared to those of the other scaffolds may be attributed to the partial destabilization of intra-molecular hydrogen bonds in collagen at $T_{\mathrm{D}}$ value of $101.96{ }^{\circ} \mathrm{C}$. However the ARG cross-linked scaffold should be intact below this temperature and this allows easy the storage at an ambient temperature for it further applications.

TGA was used to determine the thermo stability of collagenbased scaffolds. Fig. $2 \mathrm{C}$ represents the pyrolytic pattern of COLCS, COL-CS-A, COL-CS-G and COL-CS-L scaffolds. The entireweight loss process takes place in three phases. Table 2 shows the weight loss range of COL-CS, COL-CS-A, COL-CS-G and COL-CS-L scaffolds at a different temperature range. The first

Table 2 Percentage of weight loss in samples at different stage

\begin{tabular}{lllll}
\hline \multicolumn{5}{c}{$\%$ \% weight loss } \\
\cline { 3 - 5 } Sample & Peak max temp $\left({ }^{\circ} \mathrm{C}\right)$ & $50-200{ }^{\circ} \mathrm{C}$ & $200-300{ }^{\circ} \mathrm{C}$ & $300-700{ }^{\circ} \mathrm{C}$ \\
\hline COL-CS & $416.32{ }^{\circ} \mathrm{C}$ & 11.97 & 43.07 & 12 \\
COL-CS-G & $415.56{ }^{\circ} \mathrm{C}$ & 3.92 & 19.14 & 4.79 \\
COL-CS-L & $621.78{ }^{\circ} \mathrm{C}$ & 4.27 & 20.74 & 14.54 \\
COL-CS-A & $576.29{ }^{\circ} \mathrm{C}$ & 2.19 & 13.96 & 6.36
\end{tabular}

(A)

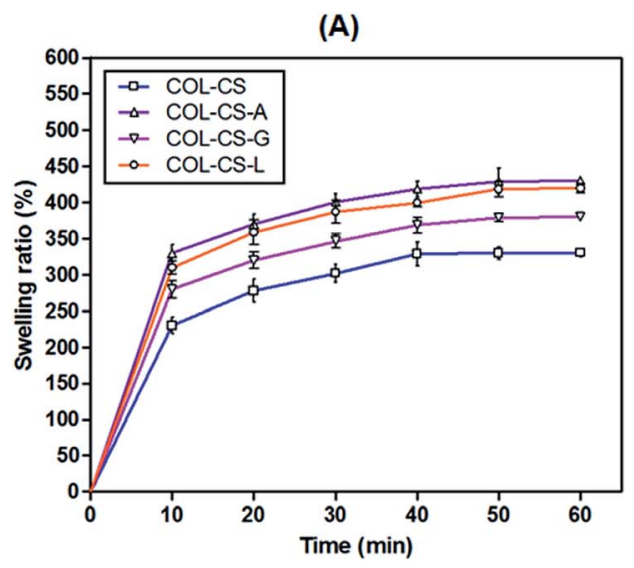

(C)

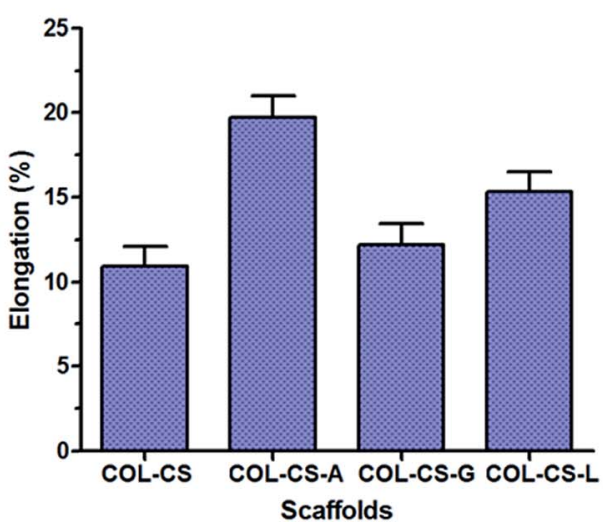
Scaffolds phase attributes to the structural water loss (dehydration) of the scaffold $\left(50-200{ }^{\circ} \mathrm{C}\right)$. All the scaffolds exhibited a significant weight loss due to the loss of free water and crystal water. The second phase results from the thermal degradation of the polymers $\left(200-300{ }^{\circ} \mathrm{C}\right)$. The COL-CS scaffold exhibited weight loss of about $43 \%$ compared to $19.14 \%$ for COL-CS-G and $20.74 \%$ for COL-CS-L, individually. However, COL-CS-A only showed $13.96 \%$ of weight loss, which is considerably lower compared to other polymeric scaffolds. The third phase refers to the carbonization of polymeric material $\left(300-700{ }^{\circ} \mathrm{C}\right) .{ }^{57}$ This finding indicates that the cross-linking of arginine leads to structural changes and may possess a stronger interaction with both collagen and chitosan molecules, thus resulting in enhanced thermal stability of the scaffold.

\subsection{Swelling studies}

The ability of a scaffold to preserve water is regarded as a swelling ability, an important characteristic to consider for tissue engineering. It can be controlled by monitoring the crosslinking density. Swelling property is influenced by several factors such as pore size, porosity, free volume and crystallinity. ${ }^{58}$ Percent swelling of COL-CS scaffold as such and its
(B)

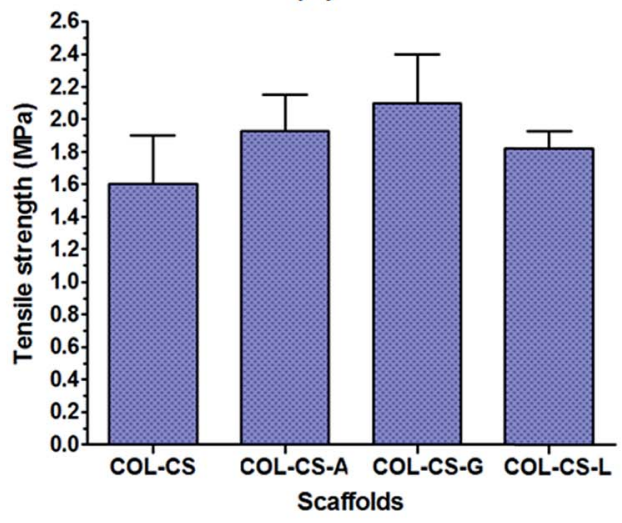

(D)

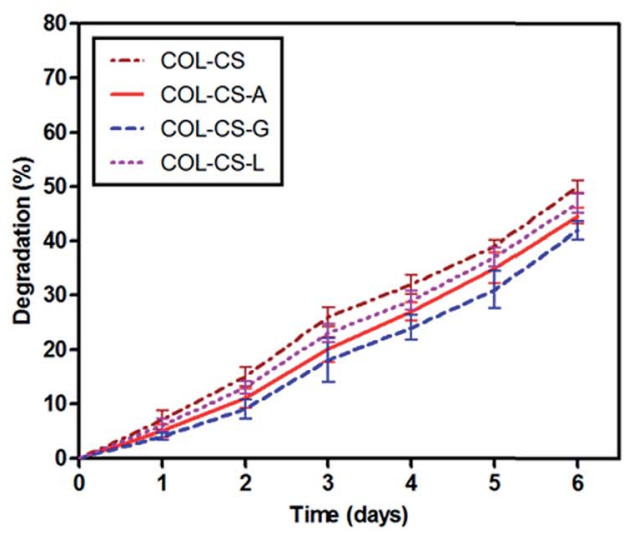

Fig. 3 (A) Swelling properties of COL-CS scaffold containing different amino acids. (B) Tensile strength, (C) elongation at break of scaffolds with and without aminoacids (COL-CS, COL-CS-A, COL-CS-G and COL-CS-L). (D) In vitro enzymatic degradation of the COL-CS scaffold without and with amino acids. Error bars represent the mean $\pm \operatorname{SD}(n=3)$. 
amino acid cross-linked forms at different intervals of water contact time are illustrated in Fig. 3A. Amino acid incorporated composite scaffolds showed higher swelling percentage compared to control scaffold. This may be due to the good interaction that exists between the amino acid and the bio polymers, which resulted in improved water holding capacity. The water uptake ability of COL-CS increased rapidly to $230 \%$ in the initial $10 \mathrm{~min}$ time interval and finally reached a saturation of $340 \%$ in $50 \mathrm{~min}$. Cross-linking of glutamic acid, lysine and arginine with COL-CS increased the swelling to 280, 310 and $330 \%$ respectively in $10 \mathrm{~min}$ and attained a saturation percent of 380,420 and $430 \%$ in $50 \mathrm{~min}$. The increase in swelling of the cross-linked biomaterial can be correlated with the number of amino/imino groups present in the cross-linking compound. The swelling rate of COL-CS-A is more than 30 times its initial dry weight, which is similar to a biological soft tissue and therefore more appropriate for tissue engineering. The water uptake ability was an indispensable property of biosupportive scaffolds that facilitated the easy diffusion of nutrients to the growing cells within the scaffolds and enhanced the absorption of culture medium. Further the swelling property allows the easy transport of nutrients and metabolites between cells and scaffold. The hydrophilic and porous structure characteristics of the scaffold can influence the water absorption, the initial adherence of the cells and increase their proliferation rate to a great extent. ${ }^{59,60}$

In this context, it is proved that the incorporation of amino acids to COL-CS considerably increased the swelling ability and hydrophilic property of the hybrid scaffold. It is also speculated that the length of the cross-linker also plays a role in

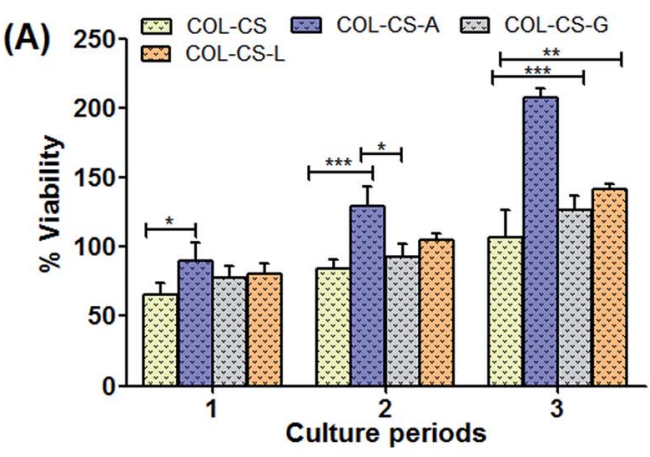

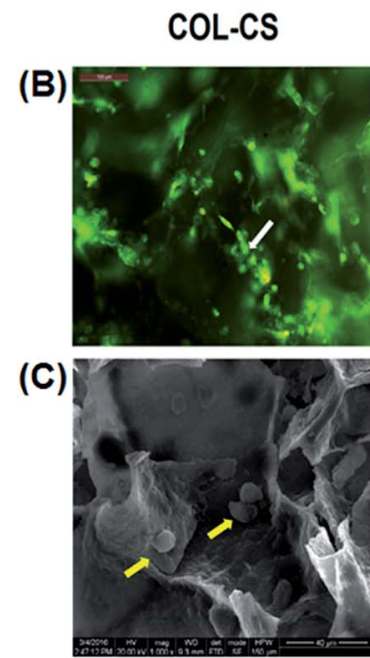

(D)

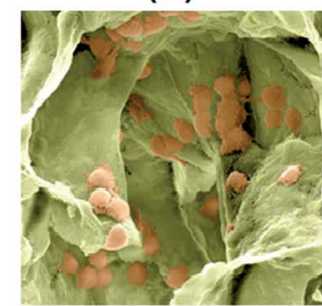

COL-CS-A
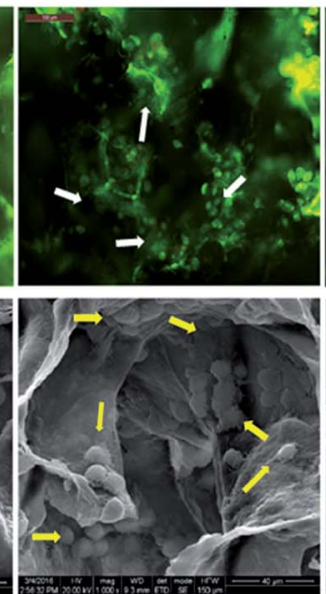

(E)

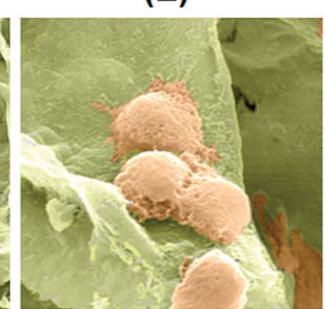

COL-CS-G
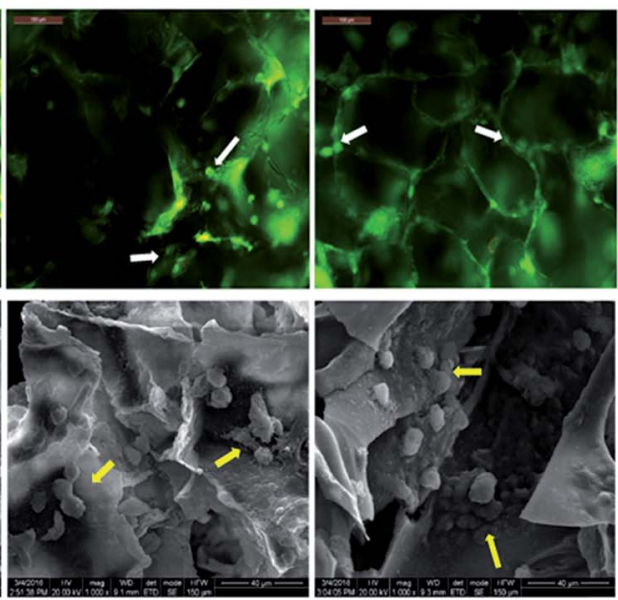

(F)

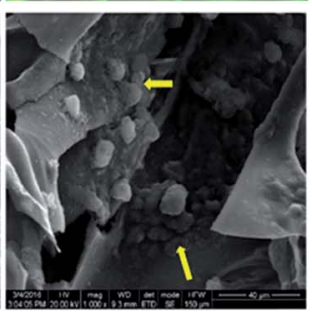

(G)
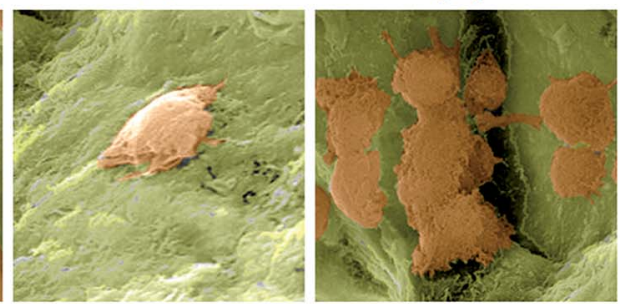

Fig. 4 MTT assay of fibroblasts cultured on COL-CS, COL-CS-A, COL-CS-G, COL-CS-L (A). Error bars represent the mean \pm SD ( $n=3$; $*$ denotes statistically significant difference, ${ }^{*} p<0.05, * * p<0.01$ and $* * * p<0.001$ ). Fluorescent images of cell-seeded 3D scaffolds were stained with AO/EB cell for morphology and proliferation studies (B), SEM images of fibroblast grown on 3D porous scaffolds on $48 \mathrm{~h}$ culture period (C), arrow indicates the fibroblast cells. Colour SEM images represents detailed view of fibroblasts spreading and interaction on COL-CS-A (D-G). 

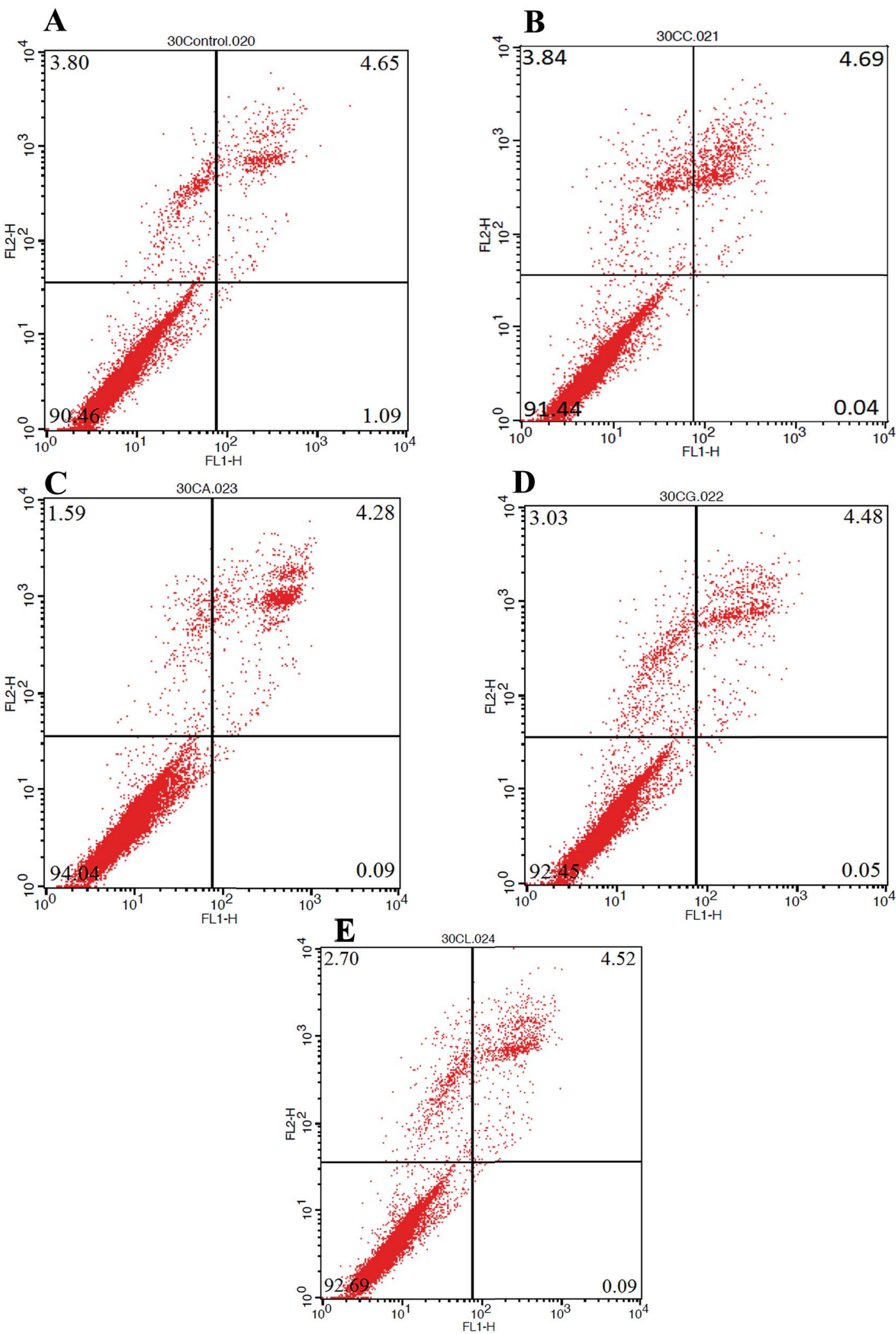

Fig. 5 FACS - FITC-labeled Annexin $V$ flow cytometric detection of apoptosis in NIH 3 T3 cells after $72 \mathrm{~h}$ incubation with the scaffolds. (A) Control, (B) COL-CS, (C) COL-CS-A, (D) COL-CS-G and (E) COL-CS-L. 
determining the water absorption of the biomaterial, as these longer amino acids set apart the macromolecules and facilitated a loose chemical structure.

\subsection{Mechanical properties}

To investigate the mechanical properties of the porous scaffolds of this study, we measured the uniaxial tensile strength in the dry condition. The tensile strength and elongation property of the COL-CS, COL-CS-A, COL-CS-G and COL-CS-L scaffolds are presented in Fig. 3B and $\mathrm{C}$. The maximum tensile strength and elongation for COL-CS scaffold and its amino acid cross-linked forms (COL-CS-A, COL-CS-G and COL-CS-L) were compared where the Young's modulus of the COL-CS scaffold was found to be $1.60 \mathrm{MPa}$ while COL-CS-A, COL-CS-G and COL-CS-L exhibited 1.93, 2.10 and $1.82 \mathrm{MPa}$ respectively (Fig. 3B). It is obvious from the results that the introduction of certain amino acids as biological cross-linker aids in increasing the tensile strength of the scaffold. The mechanical property of the individual scaffold varied depending upon the amino acid used for cross-linking. A relatively increased tensile strength exhibited by COL-CS-G compared to that of others may be due to the increased number of ionic interactions contributed by two - $\mathrm{COOH}$ groups present in the two terminals of glutamic acid. Those groups have a tendency to interact with two different $-\mathrm{NH}_{2}$ groups within chitosan or between the $-\mathrm{NH}_{2}$ of $\mathrm{CS}$ and $-\mathrm{NH}_{2}$ COL. Additionally the $-\mathrm{NH}_{2}$ of this amino acid can also interact with the $-\mathrm{COOH}$ group of collagen $-\mathrm{NH}_{2}$. Therefore, the possibility of both intra as well as inter molecular interaction by glutamic acid in the hybrid-biomolecule is very high compared to that of other two amino acids of this study. However, the COL-CS-G was highly brittle in nature with firm texture, which is attributed to increased cross-linking and therefore felt not suitable for the tissue-engineering purpose. Contrary to the tensile property, the elongation property in the case of COL-CS$\mathrm{G}$ was low due to its rigidity. Interestingly, increased elongation was measured for COL-CS-A compared to others (Fig. 5C). This 3D scaffold maintains the low strength with high extensibility. The COL-CS-L was too resilient and its scaffold pore shape was not maintained when dipped in cell culture medium, whereas the COL-CS-A scaffold displayed better stability in the medium. A higher tensile strength with a relatively better elongation would be favorable for practical handling in the tissue engineering experiments.

\subsection{In vitro biodegradation}

The in vitro enzymatic degradation profile of COL-CS scaffold before and after cross-linking with amino acids was shown in Fig. 3D. The data clearly indicate the controlled biodegradation of collagen and release of hydroxyproline in an increasing linear curve with respect to time. On the first day, COL-CS underwent 7\% degradation, whereas arginine, glutamic acid and lysine showed 5, 4 and $6 \%$ respectively. On the sixth day, the percentage of degradation of COL-CS and cross-linked scaffolds (COL-CS-A, COL-CS-G and COL-CS-L) was 50, 45, 42 and 47\% respectively. In general, the biodegradation rate of all the scaffolds was increased with immersing time. Among the cross- linked groups, COL-CS-G showed the least degradation to collagenase digestion. It is speculated that the $-\mathrm{NH}_{2}$ group of lysine enters into co-valent cross-linking with the back bone $-\mathrm{C}=\mathrm{O}$ group of intra- or inter-molecular polypeptide chains, thereby imparting stability against collagenase activity. However, in the case of arginine, though it contains the free $-\mathrm{NH}_{2}$ group at its tail end, as part of guanidine, it is likely to create steric hindrance for covalent linkage with in the triple helical molecule to its adjacent helix. These findings revealed that the incorporation of amino acids is indispensable for enhanced biostability.

\subsection{Cell viability}

The time course cytocompatibility of amino acid cross-linked scaffolds was studied and compared with COL-CS as a positive control. Fig. 4 shows the viability of fibroblasts cultured on COL-CS, COL-CS-A, COL-CS-G, COL-CS-L scaffolds for $24 \mathrm{~h}$, $48 \mathrm{~h}$ and $72 \mathrm{~h}$ culture period by MTT assay. As shown in Fig. 4A, the cell density at $24 \mathrm{~h}$ in all the scaffolds was the same; no significant difference was observed between cross-linked and plain COL-CS scaffolds. The viability of fibroblast cells in all cross-linked scaffolds were increased significantly $(P<0.01)$ with the prolongation of culture time to $48 \mathrm{~h}$. This suggests that the fibroblast efficiently anchored on the 3D scaffold, started enhancing the proliferation and migrated through the interior of the scaffold. Although, all the scaffolds were seeded with an equal number of cell density, the arginine cross-linked scaffold showed dark purple color upon addition of MTT due to the presence of increased cell density compared to other scaffolds
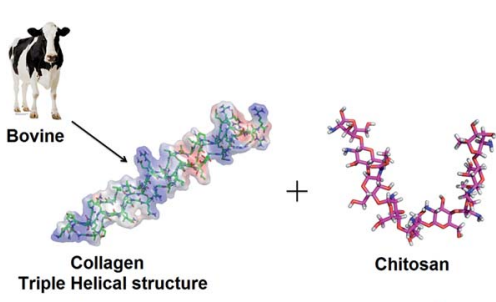

Triple Helical structure
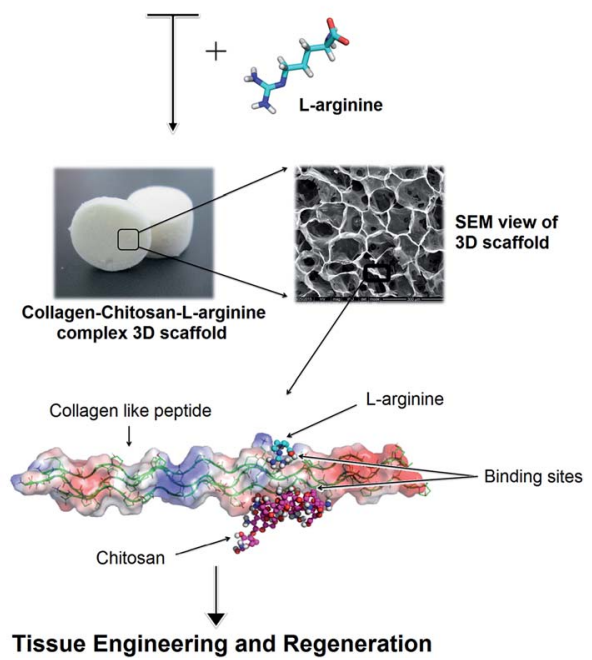

Scheme 1 Schematic representation of the mechanism involved in collagen-chitosan-L-arginine complex 3D scaffold. 
that was confirmed by OD $\lambda=540 \mathrm{~nm}$. Several studies have reported that the $3 \mathrm{D}$ scaffold provides more mechanical stability and interconnected porous network for cellular activity. ${ }^{\mathbf{4 , 6 1 , 6 2}}$ In the present study, the cell proliferation rate of different scaffolds was observed in the order: COL-CS-A $>$ COLCS-L > COL-CS-G > COL-CS. As observed from the results, 3D collagen scaffolds demonstrated their ability to increase the proliferation rate in prolonged time up to three days (72 h) without causing any toxic effect to the cells. This confirms the biocompatibility of scaffolds with or without cross-linking. Notably, COL-CS-A have shown a significant difference among all other cross-linked scaffolds, with a high degree of compatibility as denoted by increased cellular population at the end of $72 \mathrm{~h}$ of incubation.

\subsection{Cell morphology and proliferation on scaffolds}

Cell attachment in COL-CS, COL-CS-A, COL-CS-G and COL-CS$\mathrm{L}$ scaffolds and their morphology were examined by fluorescence microscopy is displayed in Fig. 4B. Fig. 4C depicts the
SEM images of fibroblast grown on 3D porous scaffolds on $48 \mathrm{~h}$ culture period. After 2 days of culture, all the four scaffolds supported fibroblast cell attachment. Fluorescent image reveals the even distribution of cells in the scaffolds, most of which were tightly adhered around the pores of the scaffold with a normal spindle shape. ${ }^{63}$ The chitosan posses cell adhesion property and it is increased when the scaffold prepared with the combination of collagen and chitosan. ${ }^{64}$ The AO/EB-stained images showed that the fibroblast had migrated through the interior of the scaffold, on the first day after cells were seeded to the scaffolds. After 2-days incubation, cells were populated at all the sides of the pores of the scaffold and were confluent. This activity was more prominent in COL-CS-A. This observation was very much in conformity with the results of the MTT assay. It was indicative that the scaffolds were able to guide the cell infiltration and proliferation, which are crucial for tissue regeneration. The SEM images (Fig. 4C) describe the spreading of the fibroblast cells on the scaffolds pronouncing the welldefined characteristics of the cytoskeleton. Cross-linked COLCS porous scaffolds permits easy penetration and adhering of

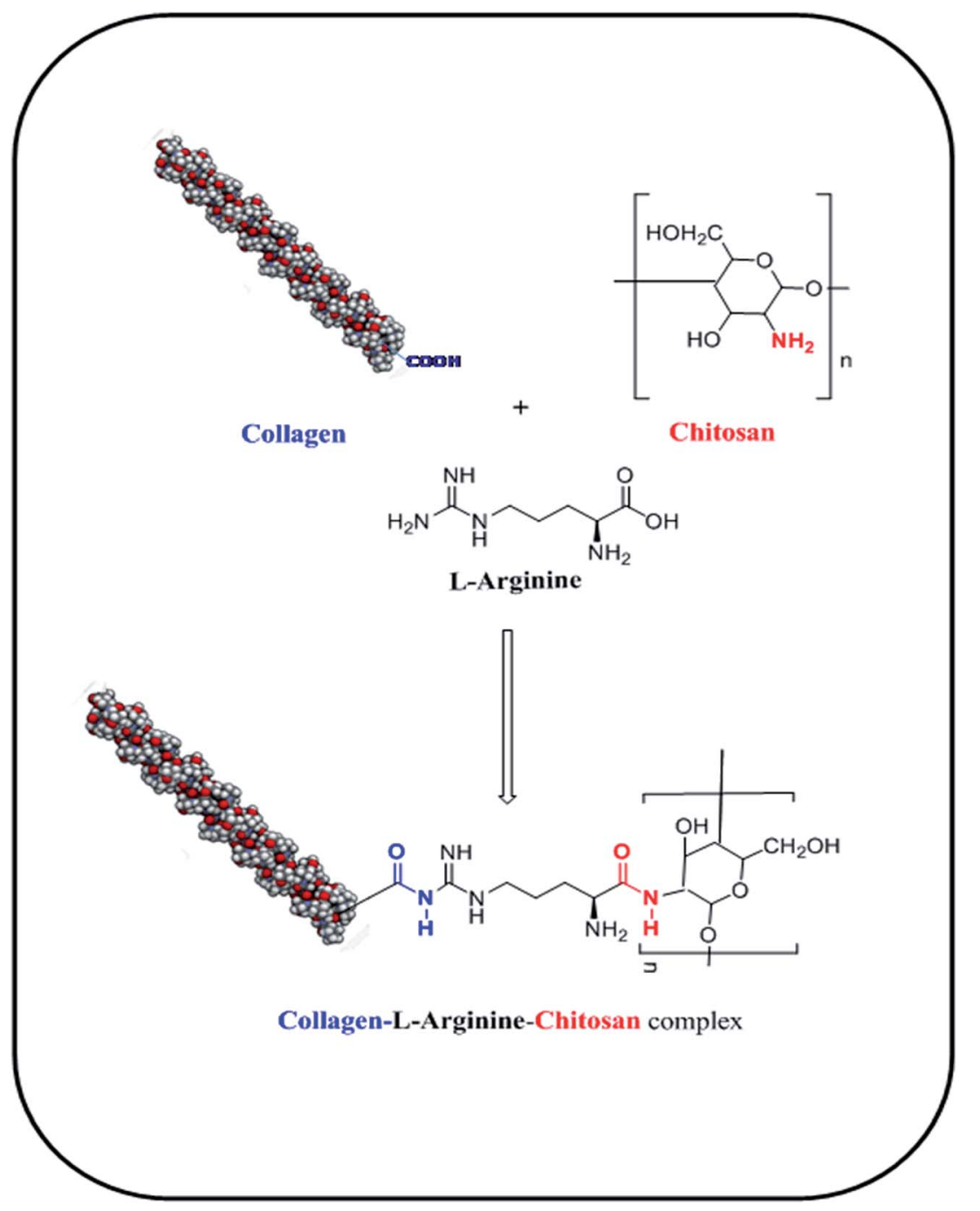

Scheme 2 L-Arginine-intercede cross-linking between collagen and chitosan molecules. 
fibroblasts onto the compartmental walls of the scaffolds. More number of cells was visualized in the amino acid cross-linked scaffolds compared to those of the COL-CS devoid of amino acid cross-linking. This ascertains the harmless quality of these amino acids as bio-cross-linkers, unlike other cross-linkers such as aldehydes. Further, the intact cell attachment, spreading and cell aggregation within pores was clearly observed in COL-CS-A scaffold (Fig. 4D). Fibroblasts make contact with their neighbours through adhesions and they connect fibroblast cells into the characteristic meshwork (Fig. 4E). Basically the fibroblast cells are predominant stromal cell type found in connective tissues and appear as plump spindle shaped or stellate shaped cells with centrally placed oval nucleus (Fig. 4F). Besides, the fibroblasts exhibit changes in the morphology and emerged as elongated cells (Fig. 4G) in the places where dense collagen fiber formation. ${ }^{65}$ Moreover, the amino acids, more particularly, the arginine, seemed to provide a harmonious environment for the cellular activity in the process of tissue engineering.

\subsection{Flow cytometry}

The cytotoxicity results obtained by MTT assay was validated by flow cytometry for detecting apoptosis or even necrosis, if any. The annexin V-FITC propidium iodide (PI) stained cells, cultured on scaffolds with or without cross-linking using different amino acids were examined for apoptosis. Healthy cells are not stained by either of the dyes and remain unaffected in their fluorescence spectrum. ${ }^{66,67}$ Fig. 5 represents the FACS FITC-labeled Annexin V flow cytometric detection of apoptosis in NIH 3T3 cells after $72 \mathrm{~h}$ incubation with the Control, COLCS, COL-CS-A, COL-CS-G and COL-CS-L. The fluorescence intensity of the cells plotted with the fluorescence of Annexin V/ FITC on the $X$-axis and fluorescence of PI on the $Y$-axis to distinguish viable cells as seen in Fig. 5 . The percentage of cell viability observed was COL-CS-A $94 \%$; followed by COL-CS-L 92.69\%, COL-CS-G 92.45\% and COL-CS 91.22\%, whereas the

Table 3 The selected best docking score collagen like peptide L-arginine and chitosan conformation based on RMSD cluster ranking and with lowest free energy resulted from AutoDock Vina

$\begin{array}{ll}\text { S. No. } & \text { Model-1 (EVGPPGPPGPAGEKGSPGADGPAGAPG) } \\ & \text { L-Arginine affinity }\left(\mathrm{kcal} \mathrm{mol}_{3}^{-1}\right) \\ 1 & -3.5 \\ 2 & -4.3 \\ & \text { Model-2 (TPGPQGIAGQRGVVGLPGQRGER })_{3} \\ & \text { Chitosan affinity }\left(\mathrm{kcal} \mathrm{mol}^{-1}\right) \\ 3 & -3.8 \\ 4 & -5.6\end{array}$

scaffold-free medium showed about $90 \%$ of viability. Though the difference in viability between the scaffolds is very narrow, COL-CS-A again pronounced its superiority over other materials in considering this for tissue engineering.

\subsection{Molecular docking of COL-CS-A complex}

The COL-CS-A system possesses better physical and biological properties; hence, the docking study was carried out to find the possible interaction of collagen with arginine and chitosan. Scheme 1 depicts schematic representation of the mechanism involved in collagen-chitosan-L-arginine complex 3D scaffold. A molecular docking result reveals that the compounds such as

Table 5 Both collagen like-peptide models residues with corresponding $\mathrm{H}$-bonds length

\begin{tabular}{llll}
\hline S. No. & Compound & Model-1 $(\AA)$ & Model-2 $(\AA)$ \\
\hline 1 & L-Arginine & GLU65-2.1 & GLN47-2.7, ARG51-2.7 \\
2 & Chitosan & GLU65-2.3 & GLU50-2.4, ARG51-1.5, GLU101-2.3
\end{tabular}
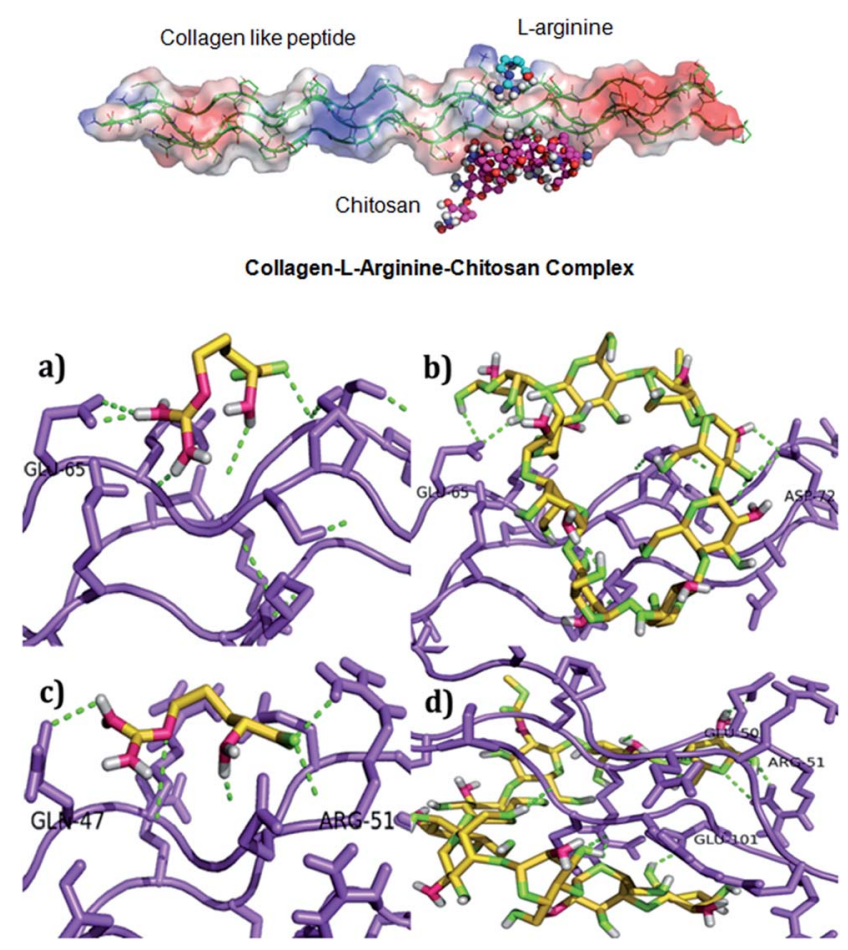

Fig. 6 The docked structure of collagen-L-arginine-chitosan complex and molecular docking conformation of (a) model-1-Larginine, (b) model-1-chitosan, (c) model-2-L-arginine, and (d) model2-chitosan.

Table 4 Collagen like peptide with L-arginine and chitosan binding involving common residues

\begin{tabular}{llll}
\hline L-Arginine & & & Chitosan \\
Model-1 & Model-2 & Model-1 & Model-2 \\
\hline GLU65 & GLN47, ARG51, GLY 49 & GLU65 & GLU50, ARG51, GLU101, ARG48, GLY49, ARG99, GLN47
\end{tabular}


chitosan and arginine bind to the charged residues of collagen like-peptide with lowest binding energy values. Table 3 shows the selected best docking score collagen like peptide t-arginine and chitosan conformation based on RMSD cluster ranking and with lowest free energy resulted from AutoDock Vina. The structural analyses of both models, like-peptide binding mode residues involved in interactions GLU 50, ARG 51, GLU 101, were actively involving interactions. Collagen like peptide with L-arginine and chitosan binding involving common residues are displayed in Table 4 . Table 5 shows both collagen like-peptide models residues with corresponding $\mathrm{H}$-bonds length. The docked structure of collagen-L-arginine-chitosan complex and molecular docking conformation of model-1-L-arginine, model1-chitosan, model-2-L-arginine, and model-2-chitosan are depicted in Fig. 6. Molecular docking reveals that the charged residues of collagen play were crucial role in interactions with arginine and chitosan.

\subsection{In vivo wound healing and tissue regeneration}

The results of in vivo wound healing on Wistar albino rats as investigated by planimetry, rate of wound contraction and percentage of healing or presented in Fig. 7A, B and C respectively. Fig. 7A shows the cutaneous excision wound, its treatment with 3D scaffold followed by closing the dressed wound by

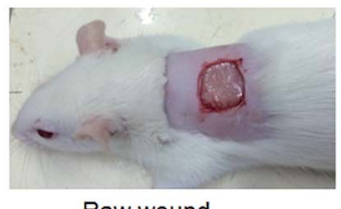

Raw wound

Day 0
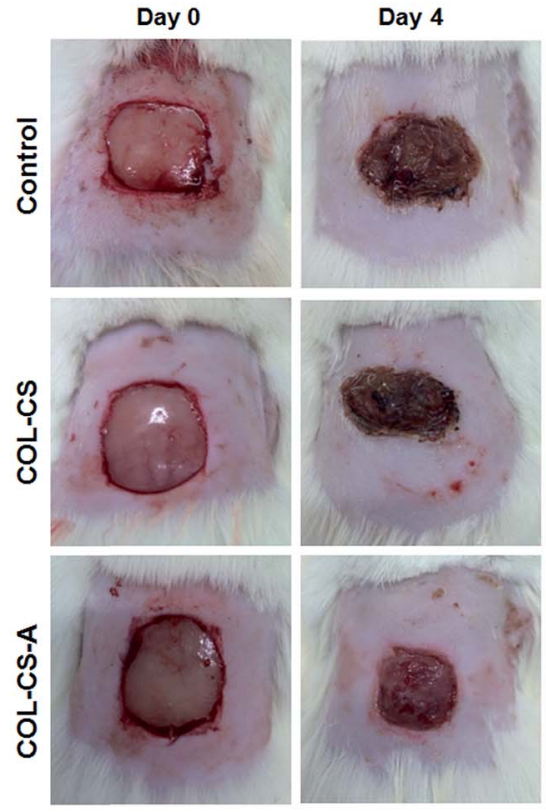

(B)

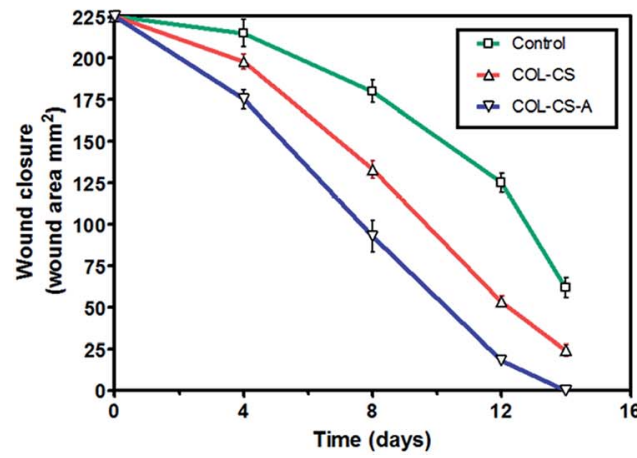

(A)

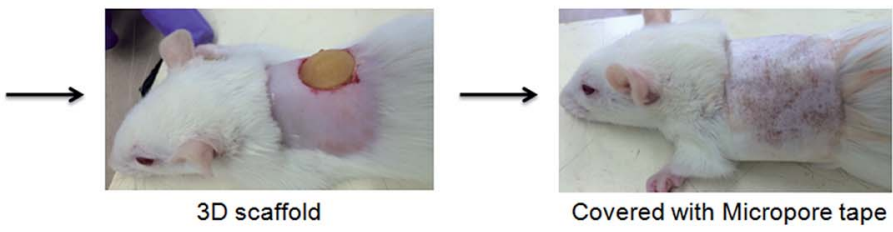

Day 12
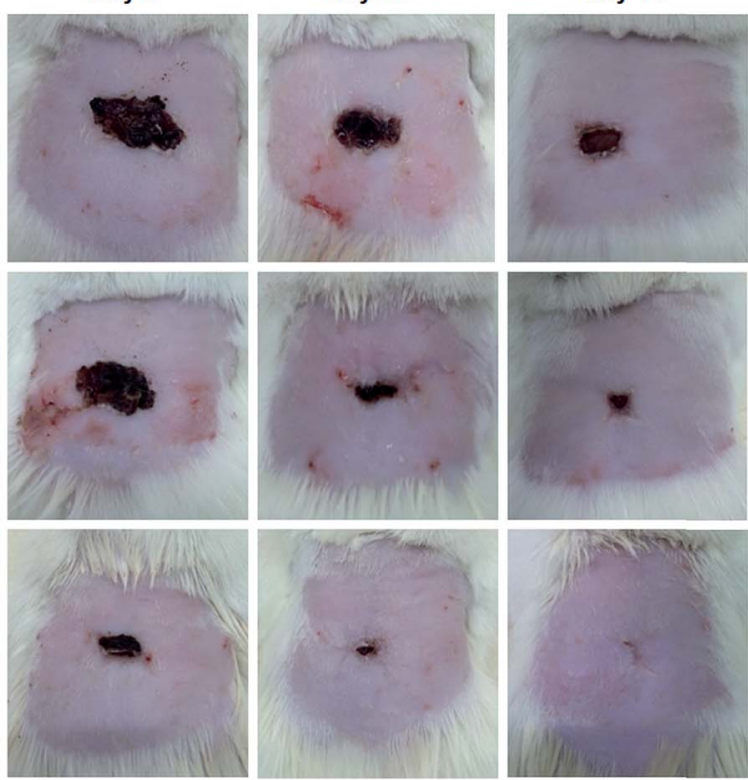

(C)

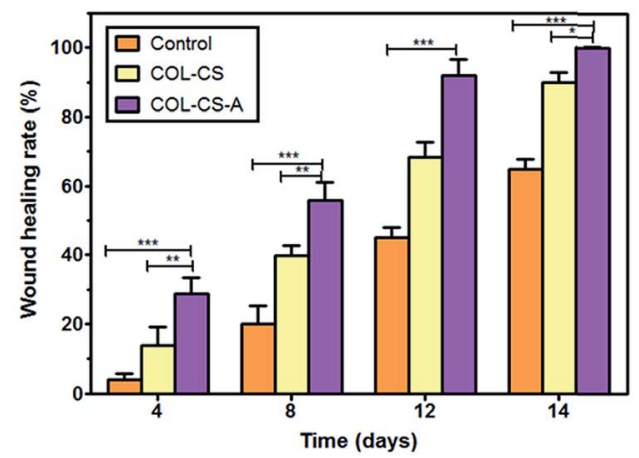

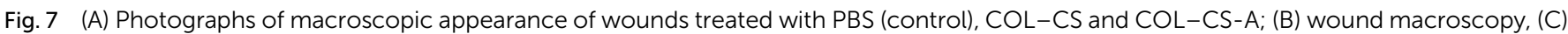

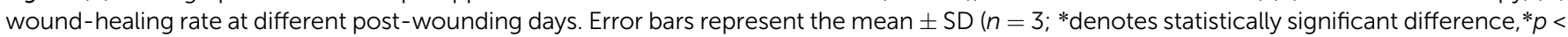
$0.05, * * p<0.01$ and $* * * p<0.001$ ). 
using micropore tape. Fig. 7B shows the periodical healing pattern of the wounds after treatment with PBS control, COL-CS and COL-CS-A dressing. The COL-CS-A scaffold dressing exhibited a good healing at the end of day 14 as against 17 and 21 days for uncrosslinked COL-CS group and control group respectively. The chitosan with collagen increase healing rate by their excellent biomimetic and chemotactic effects at the wound surface. ${ }^{43}$ Fig. 7C represents wound closure data of wounds of COL-CS, COL-CS-A and control groups. The COL-CS-A displayed an enhanced healing that could be clearly distinguished from day 4 post-wound onwards, as could be seen from the wound closure data. This was also evidenced by the reduction in the size of the wound contour, and the percentage reduction in the wound area was calculated at determined intervals of epithelialization period that was showed in Fig. 7D. After 4 days, the wound treated with COL-CS-A achieved closure up to $22 \%$, compared to COL-CS and control groups, which showed only $14 \%$ and $5 \%$ respectively. The rate of healing progressed in the same trend and showed about $92 \%$ wound closure in COL-CS-A as against $72 \%$ and $55 \%$ respectively for uncrosslinked scaffold and PBS-treated groups. The complete wound closure was observed on day 14 for COL-CS-A, while it was attained on day 17 for COL-CS and 21 days for control. The in vivo wound healing results clearly demonstrated that the porous L-arginine cross-linked COL-CS scaffold dressing could accelerate the wound-healing process, more particularly at the early stage, probably by shortening the inflammation period. The presence of arginine as a cross-linker would have also helped in

\section{(A)}

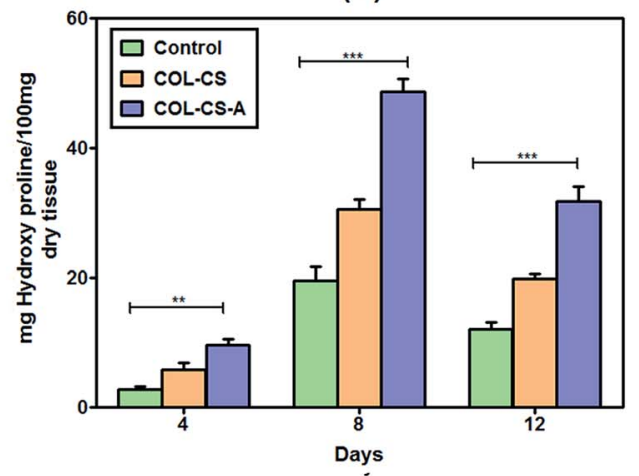

(B)

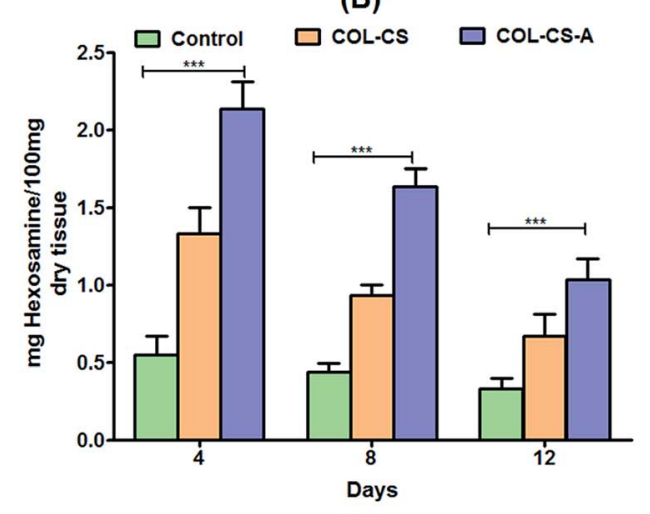

(D)

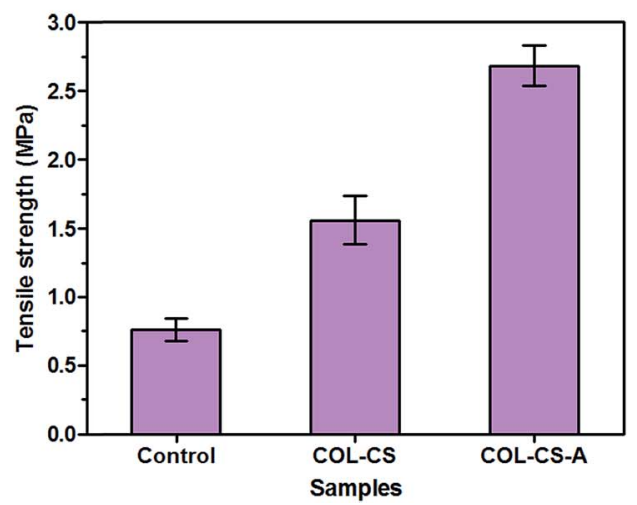

(C)

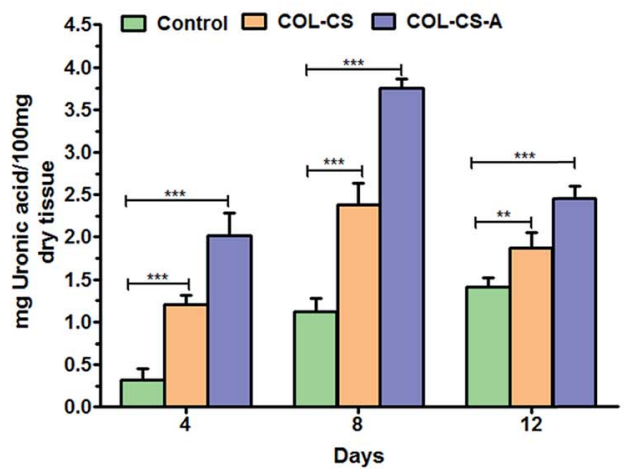

(E)

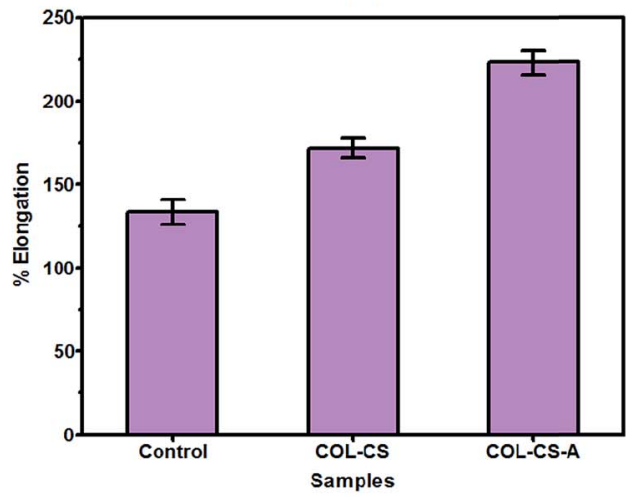

Fig. 8 Determination of (A) hydroxyproline, (B) hexosamine and (C) uronic acid content in granulation tissue and (D) tensile strength, (E) \% elongation of healed tissue. Error bars represent the mean \pm SD $(n=3$; *denotes statistically significant difference, $* * p<0.01$ and $* * * p<0.001)$. 
increasing the positively charged aminogroups in the biomaterial that would have contributed to the increased cellular attachment and proliferation on the scaffold. The increased cellular activity thus prompted in the COL-CS-A might have led to the fast healing in this group compared to the healing rate in others.

\subsection{Biochemical parameters}

The contents of the ECM constituents of granulation tissue such as collagen, hexosamine, and uronic acid in the control, COL-CS and COL-CS-A are depicted in Fig. 8. The collagen content calculated from the hydroxyproline level in granulation tissue was low on day 4 was found and increased on day 8 in all the groups.
The result implies that the collagen synthesis and its deposition on the wound surface increased due to increased fibroblast proliferation and collagen production. Fibroblasts synthesize collagen in the wound that plays a significant role in the hemostasis and structural integrity of the newly developed matrix at the wound and aids in re-epithelialization. ${ }^{68}$ Such activity was very high in the COL-CS-A group followed by COL-CS and then control. The decreased collagen concentration was observed on day 12 post-wounding in the control group that is due to a prolonged inflammatory phase. The high collagen concentration in the initial stage of healing in test groups elucidate the biocompatibility of the scaffolds that aids in the migration of fibroblasts in wound site for further collagen production.
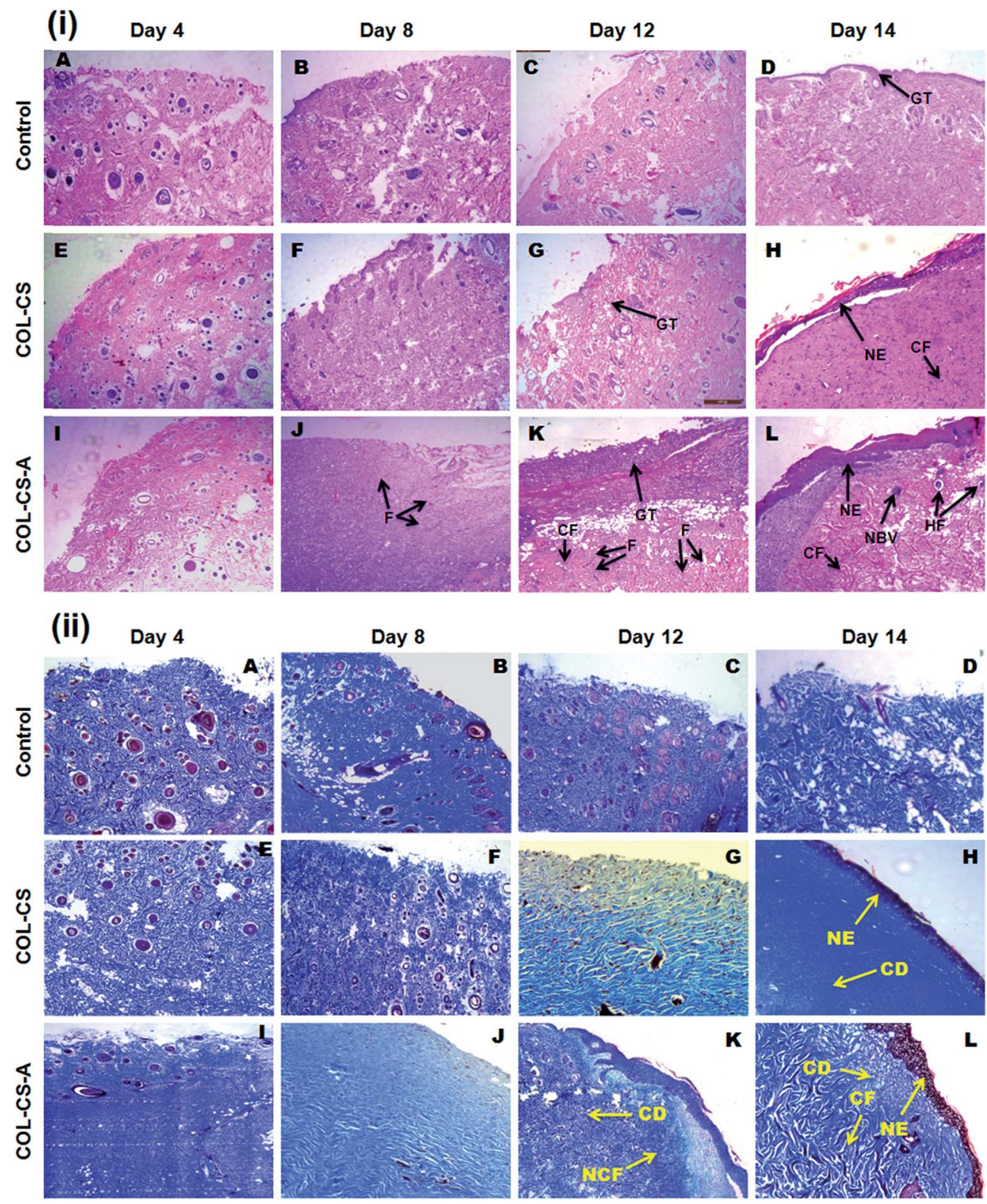

Fig. 9 Photomicrographs of wounded skin tissue of control, COL-CS and COL-CS-A group stained with (i) hematoxylin and eosin (H\&E) stain and (ii) Masson's Trichrome staining (MT) on day 4, 8, 12 and 14, 20× magnification. GT: granulation tissue, F: fibroblasts, HF: hair follicles, NBV: new blood vessels, NE: neo epidermis, CD: collagen deposition, NCF: new collagen fibers, CF: collagen fibers. 
Hexosamine is a component of ECM that serves as a nutrient for fibroblast cells to support their activity during healing. The hexosamine content in the tissue was high during the first four days post-wounding, which is considered inflammation period (Fig. 8B). Unlike collagen, the hexosamine production was found to decrease after day 4. COL-CS-A treated wounds showing a relatively higher hexosamine level throughout the experimental days followed by COL-CS and control. However, a significant difference between the test and control groups was observed. Uronic acid content in test specimen exhibited an increased trend in the order of control > COL-CS > COL-CS-A. Like collagen, the uronic acid level also significantly higher on the $8^{\text {th }}$ day in all the groups, and the trend was similar to that of collagen content (Fig. 8C). After day 12, the uronic acid level started showing the opposite trend, which is also similar to the collagen level. The decline in the concentration of hexosamine and uronic acid on day 12 is the evidence of newly formed collagen remodeling. Furthermore, the COL-CS and COL-CS-A specifically contain reconstituted type 1 collagen within their 3D matrixes, which absolutely dissolved in wound site and improved the healing rate.

\subsection{Tensile strength}

The tensile strength and percent of elongation of the skin tissue of control, COL-CS and COL-CS-A groups are presented in Fig. 8D and E. According to this data the healed tissue of the COL-CS-A group exhibited a much higher tensile value followed by COL-CS, compared to the control group. The tensile strength of the COL-CS treated was three times greater than that of the control, while the COL-CS groups displayed a five-fold increase (Fig. 8D). This difference illustrates the proportionate increase in the collagen content of the regenerated tissue. Fig. 8A already showed augmented collagen synthesis in the COL-CS group followed by COL-CS and control. Likewise, the increased tensile strength property showing the tissue specimen of COL-CS-A is justified. Like tensile strength, the elastic property of the regenerated tissue also found an increase in a 12-day postwound sample (Fig. 8E). The percentage elongation values of the samples are in the order of control $<$ COL-CS $<$ COL-CS-A which follows the trend set by the strength property. The elastic property of the tissue is determined by its uronic acid content, while the physical strength is determined by the collagen content. However, the process of the collagen fiber formation by inters and intramolecular crosslink in the remodeling phase of new collagen deposition is responsible for the entire mechanical property of the healed tissue.

\subsection{Histological observations}

The histological features of the wounded skin specimens of control, COL-CS and COL-CS-A groups on day 4, 8, 12 and 14 are shown in Fig. 9i. The appearance of immune cells after staining with H\&E, on day 4 indicates that the wounds are in the inflammatory phase. On day 8 , inflammation is considerably reduced in the COL-CS group while no inflammatory cells were found in COL-CS-A (Fig. 9i-F and J). The granulation tissue acts as a template for the neo-dermis and epithelialization

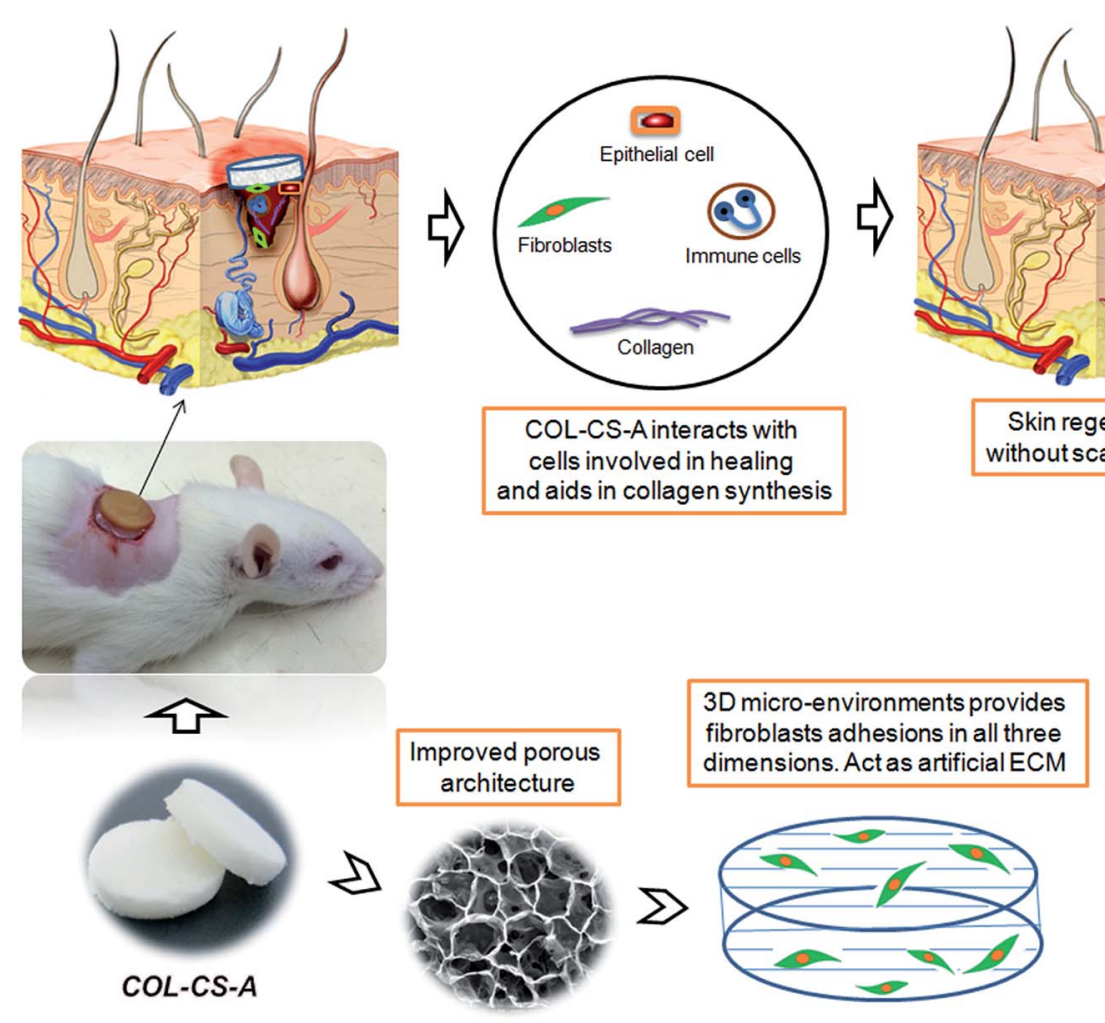

Fig. 10 Mechanism of COL-CS-A tissue engineering scaffold activated wound healing. 
formation in the wound. At day 12, a well-organized granulation tissue is observed in the test groups (Fig. 9i-G and K), while no granulation is observed in the control group. The thick collagen fibers with regular arrangements were found in COL-CS-A. These findings can be related with the wound healing rate on day 12 , which shows more than $90 \%$ of wound contraction that led to the prevention of deformity during tissue reepithelialization and remodeling. Additionally, the control group had loosely packed collagen fibers with an irregular arrangement on day 12 . Also, the incomplete epithelialization with intense fibroblast cells was seen in the control groups. The reconstructive newly formed blood vessels are clearly visible in the COL-CS-A-treated wound, which is a good sign of epithelial cell migration (Fig. 9i-L). It indicates that the L-arginine crosslinked 3D COL-CS scaffold dressing could improve the wound tissue repair and angiogenesis capabilities. In addition, at day 14 , the neo-epidermis formation can be observed in all the test groups (Fig. 9i-H and L). However, the thickness of the neoepidermis significantly varies, where a COL-CS-A group has the thickest neo-epidermis in all groups.

The neoformative fibroblasts with minimal collagen will replace the necrotic tissue at the time of injury. Fibroblasts synthesize collagen that forms the ECM matrix in the wound. Further, the collagen supports the proliferation of fibroblast and epithelial cells. Hence, it is crucial to determine the collagen deposition as wound healing progresses. ${ }^{66}$ The extent of collagen deposition was estimated by histomorphometry on tissue sections stained with MT. Fig. 9ii shows the MT staining of wound histology sections of control, COL-CS and COL-CS-A groups for day 4, 8, 12 and 14. After 12 days of healing, the collagen deposition at the COL-CS-A and COL-CS groups is much denser than that of the control, and the COL-CS-A treated wound displays the dense collagen. The light and dark blue color in (Fig. 9ii-K) indicates the loosely and tightly packed collagen fibers respectively. When compared to the control, the COL-CS and COL-CS-A have more dense and well-organized collagen fibers (Fig. 9ii-L). The control wound tissue displayed sparse and untidy collagen fibers (Fig. 9ii-D), where the wellorganized collagen fibers are found in the COLCS-A group (Fig. 9ii-L). The whole process involved in the wound treated with COL-CS-A scaffold and its action with cells for accelerated healing is depicted in Fig. 10. The results of MT staining illustrates that the COL-CS-A-treated wound tissue showed excellent tissue repair, angiogenesis capabilities and enhanced collagen deposition with well-organized fiber arrangement.

\section{Conclusion}

In conclusion, the use of amino acids - the building blocks of protein, as a cross-linker to bridge the collagen polypeptides or to link the bovine collagen and chitosan molecules imparted structural stability in the scaffold. L-Arginine proved to be a novel tool for bio-crosslinking of the natural polymers. The COL-CS-A scaffold had improved porous architecture, good swelling ability and enhanced biostability. Additionally, the amino acids provided excellent cytocompatibility due to their known biological value. Among the amino acids used, ARG plays a distinct chemical and a biological role in defining the scaffold microstructure as well as in guiding the cells on the matrix surface. Moreover, the COL-CS-A complex finds arginine as the binding pocket on the protein from the molecular docking studies. In vivo wound healing evaluation in the rat model exhibits that the L-arginine cross-linked COL-CS scaffold dressing possesses faster wound healing compared to uncrosslinked COL-CS, and it also shows excellent re-epithelialization and dense collagen fiber deposition capabilities in histological observation. The results demonstrate that the prepared COL-CS-A dressing has the potential application in chronic and diabetic wounds. The observation of this study prompts us to investigate further on the clinical trial by employing these kinds of scaffolds for tissue regeneration (wound healing) or for using this hybrid scaffold for tissue construction and transplantation.

\section{Conflict of interest}

The author(s) declare no conflicts of interest to disclose.

\section{Acknowledgements}

S. Udhaya kumar, the first author gratefully acknowledges the Indian Council of Medical Research (ICMR), Govt of India, New Delhi for financial support to this research work through Senior Research Fellowship (No. 5/3/8/2/ITR-F/2016-ITR).

\section{References}

1 L. Ma, C. Y. Gao, Z. W. Mao, J. Zhou, J. C. Shen, X. Q. Hu and C. M. Han, Biomaterials, 2003, 24, 4833-4841.

2 M. Peter, N. Ganesh, N. Selvamurugan, S. V. Nair, T. Furuike, H. Tamura and R. Jayakumar, Carbohydr. Polym., 2010, 80, 687-694.

3 N. Kawazoe, X. Lin, T. Tateishi and G. Chen, J. Bioact. Compat. Polym., 2009, 24, 25-42.

4 P. Jithendra, A. Merlin Rajam, T. Kalaivani, A. Baran Mandal and C. Rose, ACS Appl. Mater. Interfaces, 2013, 5, 7291-7298.

5 Q. J. Lu, K. Ganesan, D. T. Simionescu and N. R. Vyavahare, Biomaterials, 2004, 25, 5227-5237.

6 P. Arpornmaeklong, P. Pripatnanont and N. Suwatwirote, Int. J. Oral Maxillofac. Surg., 2008, 37, 357-366.

7 Y. Li, F. Chen, J. Nie and D. Yang, Carbohydr. Polym., 2012, 90, 1445-1451.

8 B. Song, C. Wu and J. Chang, Acta Biomater., 2012, 8, 19011907.

9 Z. Pan and J. Ding, Interface Focus, 2012, 2, 366-377.

10 G. H. Kim, S. H. Ahn, Y. Y. Kim, Y. S. Cho and W. Chun, J. Mater. Chem., 2011, 21, 6165-6172.

11 Z. Chen, X. Mo, C. He and H. Wang, Carbohydr. Polym., 2008, 72, 410-418.

12 A. Faikrua, R. Jeenapongsa, M. Sila-asna and J. Viyoch, ScienceAsia, 2009, 35, 247-254.

13 A. S. Badami, M. R. Kreke, M. S. Thompson, J. S. Riffle and A. S. Goldstein, Biomaterials, 2006, 27, 596-606. 
14 K. Sisson, C. Zhang, M. C. Farach-Carson, D. B. Chase and J. F. Rabolt, J. Biomed. Mater. Res., Part A, 2010, 94, 13121320.

15 Y. Takahashi and Y. Tabata, J. Biomater. Sci., Polym. Ed., 2004, 15, 41-57.

16 M. N. Taravel and A. Domard, Biomaterials, 1993, 14, 930938.

17 C. K. Peng, S. H. Yu, F. L. Mi and S. S. Shyu, J. Appl. Polym. Sci., 2006, 99, 2091-2100.

18 J. K. Francis Suh and W. T. Howard, Biomaterials, 2000, 21, 2589-2598.

19 M. N. Taravel and A. Domard, Biomaterials, 1996, 17, 451455.

20 S. Y. Ong, J. Wu, S. M. Moochhala, M. H. Tan and J. Lu, Biomaterials, 2008, 29, 4323-4332.

21 I. Aranaz, M. Mengibar, R. Harris, I. Panos, B. Miralles and N. Acosta, Curr. Chem. Biol., 2009, 3, 203-230.

22 C. M. Lehr, J. A. Bouwstra, E. H. Schacht and H. E. Junginger, Int. J. Pharm., 1992, 78, 43-48.

23 J. Yang, F. Tian, Z. Wang, Q. Wang, Y. J. Zeng and S. Q. Chen, J. Biomed. Mater. Res., 2008, 84, 131-137.

24 A. Di Martino, M. Sittinger and M. V. Risbud, Biomaterials, 2005, 26, 5983-5990.

25 Z. Raymond, P. J. Dijkstra and P. B. Van Wachem, Biomaterials, 1999, 20, 921-931.

26 J. E. Lee, J. C. Park, Y. S. Hwang, J. K. Kim, J. G. Kim and H. Suh, Yonsei Med. J., 2001, 42, 172-179.

27 H. Human, D. Bezuidenhout, M. Torrianni, M. Hendriks and P. Zilla, Biomaterials, 2002, 23, 2099-2103.

28 A. Maranto and F. Schoen, Circ. Res., 1988, 63, 844-848.

29 L. Ma, C. Gao, Z. Mao, J. Zhou and J. Shen, Biomaterials, 2004, 25, 2997-3004.

30 A. L. Bertone, K. E. Sullins, T. S. Stashak and R. W. Norrdin, Am. J. Vet. Res., 1985, 46, 1438-1444.

31 K. Jean Yvorchuk-St, E. Gaughn, G. St. Jean and R. Frank, Am. J. Vet. Res., 1995, 56, 1663-1667.

32 T. D. Turner, Vet. Dermatol., 1997, 8, 235-242.

33 S. F. Swaim, R. L. Gillette, E. A. Sartin, S. H. Hinkle and S. L. Coolman, Am. J. Vet. Res., 2000, 61, 1574-1578.

34 Y. J. Park, Y. Hwang, K. H. Park, J. W. Suh, D.-W. Shim, S. H. Han, J. W. Lee and W. J. Choi, Foot \& Ankle Orthopaedics, 2016, vol. 1, p. 1.

35 R. Judith, M. Nithya, C. Rose and A. B. Mandal, Biologicals, 2012, 40, 231-239.

36 D. T. Efron, S. J. Kirk, M. C. Regan, H. L. Wasserkrug and A. Barbul, Surgery, 1991, 110, 327-334.

37 S. J. Kirk, M. Hurson, M. C. Regan, D. R. Holt, H. L. Wasserkrug and A. Barbul, Surgery, 1993, 114, 155-159. 38 Z. Mohamed Gad, J. Adv. Res., 2010, 1, 169-177.

39 B. Lubec, M. Hayn, E. Kitzmuller, H. Vierhapper and G. Lubec, Free Radical Biol. Med., 1999, 22, 355-357.

40 A. Barbul, H. L. Wasserkrug, E. Seifter, G. Rettura, S. M. Levenson and G. Efron, J. Surg. Res., 1980, 29, 228-235.

41 R. S. Fishel, A. Barbul, W. E. Beschorner, H. L. Wasserkrug and G. Efron, Ann. Surg., 1987, 206, 25-29.
42 A. Barbul, Prog. Clin. Biol. Res., 1988, 266, 161-175.

43 K. Gopal Shankar, S. Udhaya Kumar, S. Sowndarya and C. Rose, RSC Adv., 2016, 6, 107403-107415.

44 I. Adekogbe and A. Ghanem, Biomaterials, 2005, 26, 72417250.

45 J. S. Pieper, A. Oosterhof, P. J. Dijkstra, J. H. Veerkamp and T. H. Van Kuppevelt, Biomaterials, 1999, 20, 847-858.

46 T. Oleg and J. Arthur Olson, J. Comput. Chem., 2010, 31, 455461.

47 P. D. Adams, Acta Crystallogr., Sect. D: Biol. Crystallogr., 2010, 66, 213-221.

48 W. Moriarty Nigel, W. Ralf, G. Kunstleve and D. Paul, Acta Crystallogr., Sect. D: Biol. Crystallogr., 2009, 65, 1074-1080.

49 M. Morris Garrett, J. Comput. Chem., 2009, 30, 2785-2791.

50 D. Seeliger and L. de. Groot. Bert, J. Comput.-Aided Mol. Des., 2010, 24, 417-422.

51 J. F. Woessner Jr, Arch. Biochem. Biophys., 1961, 93, 440-447. 52 L. A. Elson and W. T. Morgan, Biochem. J., 1933, 27, 18241828.

53 T. Bitter and H. M. Muir, Anal. Biochem., 1962, 4, 330-334.

54 L. Cyster, D. Grant, S. Howdle, F. Rose, D. Irvine, D. Freeman, C. Scotchford and K. Shakesheff, Biomaterials, 2005, 26, 697-702.

55 B. L. P. Lee, Z. Tang, A. Wang, F. Huang, Z. Yan, D. Wang, J. S. Chu, N. Dixit, L. Yang and S. Li, Acta Biomater., 2013, 9, 7264-7275.

56 Y. Zhang, W. T. Liu, G. Y. Li, B. Shi, Y. Q. Miao and X. H. Wu, Food Chem., 2007, 103, 906-912.

57 M. M. Horn, V. C. A. Martins and A. M. D. Plepis, Carbohydr. Polym., 2009, 77, 239-243.

58 L. Monfregola, V. Bugatti, P. Amodeo, S. De Luca and V. Vittoria, Biomacromolecules, 2011, 12, 2311-2318.

59 N. Mohan and P. D. Nair, Trends Biomater. Artif. Organs, 2005, 18, 219-224.

60 C. H. Kim, M. S. Khil, H. Y. Kim, H. U. Lee and K. Y. Jahng, J. Biomed. Mater. Res., 2006, 78, 283-290.

61 P. Newman, Z. Lu, S. Roohani Esfahani, T. Church, M. Biro, B. Davies, A. King, K. MacKenzie, A. Minett and H. Zreiqat, J. Mater. Chem. B, 2015, 3, 8337-8347.

62 N. Naseri, J. Michel Poirier, L. Girandon, M. Fröhlich, K. Oksman and A. P. Mathew, RSC Adv., 2016, 6, 5999-6007.

63 K. M. Hakkinen, J. S. Harunaga, A. D. Doyle and K. M. Yamada, Tissue Eng., Part A, 2011, 17, 713-724.

64 K. Gopal Shankar, S. Udhaya Kumar, S. Sowndarya, P. Suresh Babu and C. Rose, J. Biomater. Appl., 2016, 30, 780-792.

65 A. D. Doyle, F. W. Wang, K. Matsumoto and K. M. Yamada, J. Cell Biol., 2009, 184, 481-490.

66 A. Ishaque, M. Al-Rubeai and R. Portner, Animal Cell Biotechnology Methods and Protocols, Humana Press, 2007, vol. 24, pp. 285-299.

67 Y. Yuan, C. Liu, J. Qian, J. Wang and Y. Zhang, Biomaterials, 2010, 31, 730-740.

68 K. Rydell-Tormanen, K. Andreasson, R. Hesselstrand, J. Risteli, D. Heinegard, T. Saxne and G. WestergrenThorsson, Lab. Invest., 2012, 92, 917-925. 